\title{
AUTONOMOUS TRACKING AND FOLLOWING OF SHARKS WITH AN AUTONOMOUS UNDERWATER VEHICLE
}

\author{
A Thesis \\ Presented to \\ the Faculty of California Polytechnic State University \\ San Luis Obispo
}

\author{
In Partial Fulfillment \\ of the Requirements for the Degree \\ Master of Science in Computer Science
}

by

Esfandiar Manii

May 2012 
(c) 2012

Esfandiar Manii

ALL RIGHTS RESERVED 


\section{COMMITTEE MEMBERSHIP}

TITLE:

AUTONOMOUS TRACKING AND FOLLOWING OF SHARKS WITH AN AUTONOMOUS UNDERWATER VEHICLE

AUTHOR: $\quad$ Esfandiar Manii

DATE SUBMITTED: $\quad$ May 2012

COMMITTEe CHAIR: Christopher M. Clark, Ph.D.

COMMITTEE MEMBER: Franz Kurfess, Ph.D.

COMMITTEE MEMBER: Zoë Wood, Ph.D. 


\begin{abstract}
AUTONOMOUS TRACKING AND FOLLOWING OF SHARKS WITH AN AUTONOMOUS UNDERWATER VEHICLE
\end{abstract}

\author{
Esfandiar Manii
}

This thesis presents the integration of an acoustic tracking system within an autonomous underwater AUV (AUV) to enable real-time tracking of sharks tagged with artificial acoustic sources. The tracking system consists of two hydrophones and a receiver unit that outputs a measurement of the relative angle to the tagged shark. Since only two hydrophones are used, the sign of the relative angle measurement is unknown. To overcome this ambiguity, a particle filter algorithm was developed to estimate the position of the acoustic source. When combined with an active control system that drives vehicle to obtain different orientations with respect to the acoustic source, real-time autonomous localization, tracking, and following of a tagged shark is shown to be possible. Four types of ocean experiments were used to validate the system including: 1) AUV tracking of a stationary tag, 2) AUV tracking of a tagged kayak, 3) AUV tracking of a tagged AUV, and 4) AUV tracking of a tagged shark. These experiments were analyzed with respect to the localization error, associated error variance, and distance between the AUV and the tag. The final shark tracking experiments took place in SeaPlane Lagoon, Los Angeles, CA, where the AUV was able to autonomously track and follow a tagged Leopard Shark for several hours. 


\section{Acknowledgements}

First, I want to acknowledge my graduate advisor, Dr. Christopher M. Clark, for his professional attitude and encouragements to the shark tracking team. Second, I would like to show my gratitude to my family who has continued to show me kindness and love throughout my life. Finally, I want to recognize the contribution of National Science Foundation for funding this project and helping our team to perform this research. 


\section{Contents}

List of Tables $\quad$ ix

List of Figures $\quad x$

1 Introduction 1

1.1 Autonomous Underwater Vehicle (AUV) . . . . . . . . . . 2

1.2 Current Research in Underwater Tracking . . . . . . . . . . . 4

1.3 Why Sharks? . . . . . . . . . . . . . . . . . 6

1.4 Current Methods of Shark Tracking . . . . . . . . . . . 7

1.5 Objectives of Shark Tracking Research _ . . . . . . . . . . 9

1.6 Objectives of the current research . . . . . . . . . . . 9

2 Mobile Tracking System Design 11

2.1 System Design . . . . . . . . . . . . . . . . . . . . . . . 11

2.1.1 Design Constraints . . . . . . . . . . . . . . . . 11

2.1.1.1 Average Sharks Speed . . . . . . . . . . . . 12

2.1.1.2 Sharks Maneuverability . . . . . . . . . . . 12

2.1.1.3 Sharks' Sensing Range . . . . . . . . . . . . . 18

2.1.1.4 Necessity of Autonomy . . . . . . . . . . . . . 19

2.1.1.5 Tracking Device Response Time . . . . . . . . . . 19

2.1.2 Design Criteria . . . . . . . . . . . . . . . . . . . . . 19

2.1.2.1 Tracking Speed . . . . . . . . . . . . . . . . . 19

2.1.2.2 System Maneuverability . . . . . . . . . . . 20

2.1.2.3 Tracking Device Response Time and Detection Range . . . . . . . . . . . . . . . 20 
2.1.2.4 Duration of System in Hours . . . . . . . . . 20

2.2 Proposed Solution . . . . . . . . . . . . . . . . . . . . . 22

2.2.1 Vehicle for Tracking . . . . . . . . . . . . . . 22

2.2.2 Device for Tracking . . . . . . . . . . . . . 23

2.2.2.1 Acoustic Tracking Device . . . . . . . . . 23

2.2.2.2 GPS Tracking Device. . . . . . . . . . 25

2.2.2.3 Visual Tracking Systems . . . . . . . . . . . 27

2.2.3 System Design Decision . . . . . . . . . . . . . 28

2.2.3.1 Final Design Decision _. . . . . . . . . . 28

2.2.4 Current Research AUV . . . . . . . . . . . . . . . . 29

2.2.5 Acoustic Tracking System, LOTEK . . . . . . . . . . . . . 32

2.2.6 Shark Tracker System Components . . . . . . . . . . . 35

2.2.6.1 Hydrophone Mounting Frame . . . . . . . . . . 36

2.2.6.2 Yaw Fin ................ 37

3 Control System 39

3.1 Control System Design . . . . . . . . . . . . . . . . . . 39

3.1.1 Cost Function Definition . . . . . . . . . . . . . 41

3.1.2 Constraints for Modeling . . . . . . . . . . . . . . . . 41

3.1.3 Assumptions. . . . . . . . . . . . . . . . . . . 42

3.2 Proposed Solution - A Bearing Modulation Controller . . . . . . . 43

3.2.1 Step Control System . . . . . . . . . . . . . . 44

3.2.2 Control System Definitions . . . . . . . . . . . . . . . . 45

3.2.3 Shark Tracker . . . . . . . . . . . . . . . . 47

3.3 Simulations . . . . . . . . . . . . . . . . . . 48

3.3.1 Autonomous Tracking System Simulation . . . . . . . . . . 50

3.3.1.1 AUV Simulation ............. 50

3.3.1.2 LOTEK System Simulation . . . . . . . . . 51

3.3.2 Standard Deviation . . . . . . . . . . . . 51

3.3.3 Simulation Data for an Improved Control System . . . . . 52

3.3.4 Setting Controller Parameters . . . . . . . . . . 53 
4 Experiments Description and Results $\quad 57$

4.1 Stationary Tag Tracking . . . . . . . . . . . . . . . . . 57

4.2 Moving Tag Tracking . . . . . . . . . . . . . . . . . 59

4.3 Secondary AUV Tracking . . . . . . . . . . . . . . . 60

4.4 SeaPlane Lagoon Field Experiments . . . . . . . . . . . . . . . . . 60

4.5 Results........................ . . 62

4.5.1 Stationary Tag Tracking . . . . . . . . . . . 62

4.5.2 Tagged AUV Tracking .............. 63

4.5.3 Shark Tracking . . . . . . . . . . . . . . . 65

5 Conclusion $\quad 69$

6 Future Works $\quad 71$

$\begin{array}{ll}\text { Bibliography } & 72\end{array}$ 


\section{List of Tables}

2.1 Tracking System Design Constraints and Criteria . . . . . . . . 21

2.2 Final Design Decision Table . . . . . . . . . . . . . . . . 30

2.3 The Autonomous Underwater Vehicle Specifications . . . . . . . . 31

2.4 Average Thrust Force Needed for Each Mounting Frame on Forward Direction . . . . . . . . . . . . . . . 37

2.5 Average Thrust Force Needed for Each Mounting Frame on Backward Direction . . . . . . . . . . . . . . . 37

2.6 Time Elapsed for the AUV to Complete a Turn with Carbon Fiber Mounting Frame and Different Yaw Fins . . . . . . . . . . 38

3.1 Standard Deviation of Particles Clusters Reduction Slopes, for the first 20 seconds . . . . . . . . . . . . . . . 56

3.2 Standard Deviation Particles Clusters Reduction Slopes, after the first 20 seconds . . . . . . . . . . . . . . . . . 56

4.1 Relative Location between AUV and Tag . . . . . . . . . . . 59

4.2 Relative Location between AUV and Tagged Kayak . . . . . . . 59 


\section{List of Figures}

1.1 Number of shark attacks in the United States from 2000 to 2010. Cited from Florida Museum of Natural History, International Shark Attack File .................. 7

2.1 Vehicle Maneuverability Analysis . . . . . . . . . . . . . 13

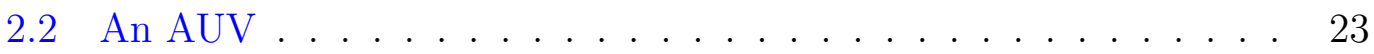

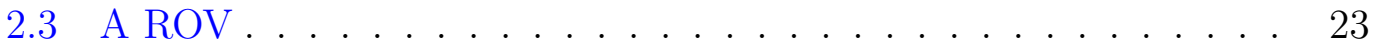

2.4 An Acoustic Tracking Device . . . . . . . . . . . . . . . . . 25

2.5 An Example of a Satellite Image . . . . . . . . . . . . . . . 27

2.6 An Underwater Video Camera Used by Visual Tracking Devices . 28

2.7 The Autonomous Tracking System Design Decision Diagram . . . 29

2.8 Autonomous Underwater Vehicle Used in the Current Research . 29

2.9 System Diagram of the AUV . . . . . . . . . . . . . . . . 32

2.10 Acoustic Tracking Device, LOTEK MAPHOST-RTA . . . . . . . 33

2.11 Acoustic Transmitter . . . . . . . . . . . . . . . . . . 34

2.12 Acoustic Tracking Device Integrated with the AUV . . . . . . . . 34

2.13 Control System Diagram of the AUV . . . . . . . . . . . . . 35

2.14 The Rig Used to Fix the Hydrophones under the AUV . . . . . . 36

2.15 Original Yaw Fin . . . . . . . . . . . . . . . . . . . . 37

2.16 New Yaw Fin .................... 37

3.1 Shark Tracking Coordinate System . . . . . . . . . . . . . . . 40

3.2 Particles Convergence . . . . . . . . . . . . . . 44

3.3 Control System Behavior . . . . . . . . . . . . . . . . . 46 
3.4 Control System Algorithm Diagram . . . . . . . . . . . . . . . 49

3.5 Graphs of Plotting the Particles Standard Deviation vs. Time with Different Yaw Angle Values . . . . . . . . . . . . . . . . 54

3.6 Setting Controller Parameters Algorithm . . . . . . . . . . . 55

4.1 Acoustic Transmitter Attached to a Buoy . . . . . . . . . . . 58

4.2 Cal Poly Pier, Avila Beach, CA. . . . . . . . . . . . . . 58

4.3 Transmitter Attached to The Second AUV . . . . . . . . . . . . 60

4.4 SeaPlane Lagoon, Los Angeles, CA. . . . . . . . . . . . . . . 61

4.5 A Leopard Shark . . . . . . . . . . . . . . . . . . . . 61

4.6 Cost Function, Error, Standard Deviation, Cost Function First Derivative, and LOTEK Signal Rate from Tracking a Stationary

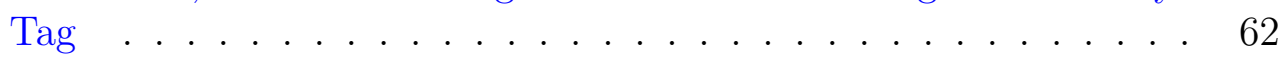

4.7 Error, Standard Deviation, and LOTEK Signal Rate from Tracking a Tagged AUV . . . . . . . . . . . . . . . . 64

4.8 Cost Function, Cost Function First Derivative, and LOTEK Signal Rate from Tracking a Tagged AUV . . . . . . . . . . . . . . 64

4.9 Close-up of the Follower AUV, the Secondary AUV, and Estimated Position Trajectories . . . . . . . . . . . . . . . . 65

4.10 Cost Function, Standard Deviation, Cost Function First Derivative, and LOTEK Signal Rate from Tracking a Tagged Shark . . . 67

4.11 AUV Yaw Angle and Bearing to the Tag from Tracking a Tagged Shark in One Control Sequence . . . . . . . . . . . . . . 67

4.12 Tagged Shark Velocity . . . . . . . . . . . . . . . . 68

4.13 Live Shark Path . . . . . . . . . . . . . . . . . . . . . 68 


\section{Chapter 1}

\section{Introduction}

For years the underwater world has attracted scientists to explore and conduct research in many areas, including: Biological Sciences, Naval Engineering, Mechanical Engineering, etc. Most of this work directly requires humans to engage and as a consequence there is an increase in human-work time and costs. Through the development of AUVs, efforts have been undertaken to reduce those costs and increase productivity in all aspects. AUVs have been used by scientists for a variety of applications, e.g. pipe monitoring, exploring, etc. One application area where AUVs have seen little use but have great potential is the study of fish movement patterns.

Throughout the world, acoustic tracking systems have been used for studying several species of fish. In most studies, the fish are caught, tagged and then released. The tags have an omnidirectional acoustic transmitter that broadcast signals at a constant rate. Receivers are used to detect and interpret the signals. Through this process, the bearing and sometimes distance to the fish can be measured. The main purpose of this thesis was to increase the mobility of such a tracking system which typically is stationary. The goal is to design an 
autonomous mobile shark tracking system for long term missions with admissible accuracy and consistency. This chapter presents some of the current research in tracking sharks using underwater AUVs and answers why autonomous shark tracking is necessary.

\subsection{Autonomous Underwater Vehicle (AUV)}

Autonomous Underwater Vehicles (AUVs), are vehicles that can operate without human aid. The main reason for designing such AUVs is to conduct underwater operations in remote or dangerous situations. AUV can operate missions that a human is not able to perform. A very simple AUV consists of two main units: 1) Navigation system, and 2) Propulsion system. The navigation system (which is autonomous) calculates the waypoints that the AUV must follow based on its program. Then it supplies the proper velocity and angle to the propulsion system to follow those waypoints. The first AUV was developed in the Applied Physics Laboratory at the University of Washington in 1957, by Stan Murph, Bob Francois and later improved by Terry Ewart. The "Special Purpose Underwater Research Vehicle", or SPURV, was used to study diffusion, acoustic transmission, and submarine wakes [1]. Currently, numerous operations are being done by AUVs. They range in size based on the operation(s) they might perform. AUVs are mostly used in the following areas: 1) Marine Biology, 2) Oceanography, 3) Commercial Purposes, and 4) Military Purposes [16]. Research mostly focuses on localization and mapping of the underwater environment. AUVs have many advantages in comparison with other types of underwater robots, (e.g. ROVs). These advantages include: 1) Navigational and positioning accuracy,

2) Survey flexibility, 3) survey time, 4) data quality and digital quality, 5) Large 
data collection, 6) Longer duration of operations, 7) Ability to perform in extreme conditions. However, AUVs encounter several problems including ocean currents and waves which may move AUVs to undesirable locations. AUV navigation uses a variety of sensors and sensor fusion techniques. A common sensor found on almost all AUVs is a GPS receiver. Since water is roughly 1000 times denser than air, electromagnetic signals cannot pass through as easily as through air and therefore diminish much faster. As a result, GPS signals can only be useful when the AUV is on the water surface or in shallow water. While underwater, several acoustic positioning systems can be used for AUVs including Conventional Long Baseline (LBL), Short Baseline (SBL), or Ultra Short Baseline (USBL) systems which are now being offered as combined systems. The unique LBL (least squares adjustment of lines of positions) or USBL (phase correlation to generate wave vectors) solutions then have to be combined with external sensor data to provide the adjusted position [53]. On the other hand, optical analysis, which consists of color, texture, shape and dynamic properties of the environment is used to analyze the image sequences for target tracking by Autonomous Underwater Vehicles (AUVs) $[9,10]$. Here, an Inertial Navigation System (INS) which improves autonomous underwater vehicle navigation for undersea explorations is investigated [27]. Also the strategic and tactical applications for autonomous submersibles place great demand on the platforms' passive sonar signal and data processing abilities. It is necessary to overcome the limited acoustic aperture and lack of human supervision by exploiting synergism between front-end signal processing functions and back-end data fusion algorithms [24]. Other types of experiments include object tracking in the underwater environment which will be discussed in the next section. 


\subsection{Current Research in Underwater Tracking}

Underwater tracking operations are typically accomplished by manually tracking instruments, at piers, or by satellites. To track fish, two methods are widely used: 1) Acoustic tracking, and 2) GPS tracking. Acoustic tracking systems are one of the most popular research tools based on other studies [22, 30, 23, 56]. These systems let researchers track fish with less labor intensive activities [22], but still require human operations to simultaneously track signals from the tags [47]. In recent years, studies have benefited from several advances in acoustic transmission systems. These studies have mainly focused on how to design arrays of hydrophones to monitor underwater habitats $[20,46,22,56,18]$ as well as gaining data from the environment and the animal using triangulation and trilateration techniques $[19,39,6]$. Most of the developments were based on research conducted using fixed hydrophones in the experimental areas. Furthermore, those technologies led to new era of mobile acoustic tracking where fixed hydrophones were placed on a boat or ship to track animals [57]. GPS tracking systems works different than acoustic tracking systems. Tracking systems based on GPS technology were initiated in the 1990s to acquire the regions in which terrestrial and Volant animals live [40, 43]. Utilizing GPS technology has some advantages over other methods due to: 1) High spatial accuracy and temporal resolutions, 2) Capacity to collect large databases about environments, 3) Ability to localize without human aid, and 4) Ability to locating any individuals. The only disadvantage is that GPS technology requires animals to swim close to the water surface. When an animal descends below the surface, tracking operations fail due to signal loss [54]. To localize and track sharks several methods can be

manipulated: e.g. 1) Stationary acoustic receivers are spread in a specific area to track sharks at any time, and 2) Boats can patrol the sea to find a tagged shark 
and to follow that shark's movements. These solutions are labor intensive and some species are lost for long periods of time.

Currently, autonomous underwater vehicles (AUVs) are well known for their high efficiency and accuracy. [32] Addresses the reason to operate AUVs for underwater explorations and research. AUVs have the potential to revolutionize our access to the oceans to address critical problems facing the marine community. Efforts to design and implement control systems and algorithms for tracking objects in dynamic environments have been done before. These methods suggest a probabilistic data association filtering to track the moving objects with mobile AUVs, [45, 44, 33], that can be extended to the underwater environment. Tracking objects in underwater environments using acoustic systems has been known as a good way of localization [2]. Some studies have been done to track Sperm Whales with AUVs by using two arrays of hydrophones to auscultate the signals from them for tracking and localization [50, 51]. Because of imperfections in vehicle control, it is common for an AUV to undergo significant yaw and pitch oscillations. Therefore for localization of underwater targets, methods have been developed to reduce the imperfections in vehicle control using sonars. These methods incorporate bearing stabilization algorithms that were implemented to address these issues on an intelligent AUV sonar sensor and tested during a subsequent sea trial with the goal of providing target bearing estimates [25]. Since imperfections can be solved, studies have been done to increase the range of acoustic signals to be used by an AUV resulted in range enhancement of acoustic devices [38]. Numerous studies also have been done to record the sounds of the underwater mammals in order to inspect their lives [26]. None of the previous studies have utilized an AUV integrated with acoustic transmission system. Also, shark tracking operations have never been performed by AUVs equipped 
with acoustic devices. The main difference between this thesis and previous research is to use an acoustic system in non-stationary states attached to an AUV to track any type of fish species underwater.

\subsection{Why Sharks?}

Based on the information provided by ISAF "International Shark Attack File" [11], every year sharks attack fatally and non-fatally. Although sharks threaten human life, sharks must also be protected. White Sharks are threatened with extinction. The number of White Sharks has been reduced in the past years and biologists are worried about their future. If the migration path of sharks and their behavior became clear to scientists, dangerous locations close to the shore would be recognized and the number of attacks close to beaches could be reduced. To perform this important task, scientists need to understand shark behavior in order to answer complicated questions including: Which habitats do sharks prefer? What is the size of their home ranges? Do White Sharks show fidelity to particular sites, such as hunting grounds, and if so for how long? How far do White Sharks travel? Do White Sharks have regular migrations, and if so what are the sizes and routes of these migrations? What are the relationships between great White Sharks that live in different parts of the world? Are the movements of White Sharks driven by environmental factors? If so, which are the most important of them? Therefore, autonomous shark tracking can be useful for two purposes: 1) Tracking for attack prevention, and 2) Extinction prevention. AUVs track sharks to study their behaviors. 


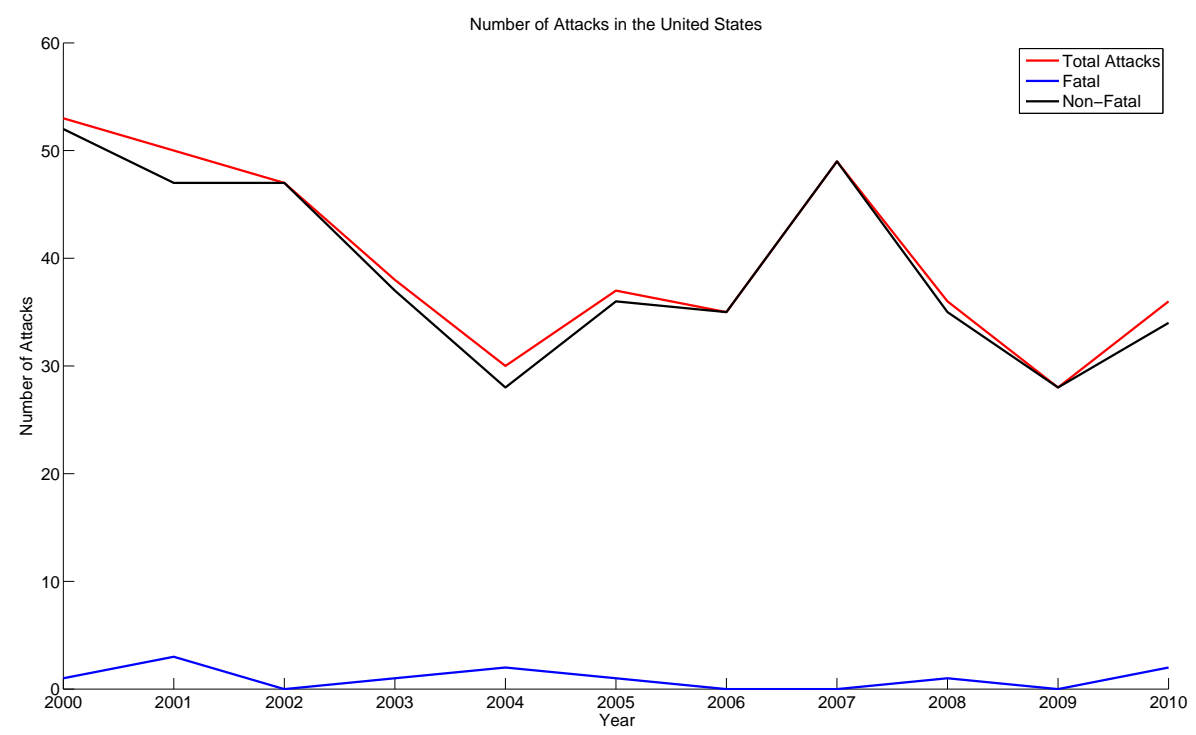

Figure 1.1: Number of shark attacks in the United States from 2000 to 2010. Cited from Florida Museum of Natural History, International Shark Attack File

\subsection{Current Methods of Shark Tracking}

One of the main reasons sharks are such effective predators is their keenly attuned senses. Initially, scientists thought of sharks as giant swimming noses. When researchers plugged the nasal openings in captive sharks, the sharks had trouble locating their prey. This seemed to demonstrate that the shark's other senses were not as developed as the sense of smell. Further research demonstrated that sharks actually have several acute senses, but that they depend on all of them working together. When you take one away, it significantly hinders the shark's hunting ability [5]. Therefore by providing a large amount of blood in the environment, sharks can be easily distracted and lured to the bait. Besides this basic method to track a shark, other methods are being used widely in research such as satellite and acoustic tracking.

In one common tracking method a near-real time tag is attached to a shark. 
When the shark swims in shallow water or gets close to the water surface, the tag sends the signal to the satellite and by getting those signals the shark can be located [19]. Another method for tracking fish is to attach an acoustic transmitter to the shark. Often, scientists are required to follow the signals from the tag by acoustic receivers.

In [18], the authors used an AUV to track two tagged Atlantic Sturgeon in the Hudson River. Results of this research proved that the AUV is capable of collecting data from a tagged fish. The AUV in this research sweeps across a specific area to get signals from the fish. Then by getting more signals, it locates the fish. Finally, in [17], the authors incorporate hydrophones receivers in an AUV. Also they validated the effectiveness of the hydrophones in the area of deployment.

In one approach, [60], the authors used an acoustic system to locate a tagged Shovelnose shark. They set up acoustic receivers in a bay and collected data when the shark came into the range. The data was analyzed to investigate the shark's behaviors based on its motion patterns. In this thesis, the focus is on using acoustic tags. This method involves tagging a shark with an acoustic device which propagates information on regular time sets.

Other methods are currently used for object tracking by robots. In [57], visual tracking systems were investigated for tracking multiple human inside a building. In this research, visual tracking is used in order to track multiple human inside a building. The authors were able to reliably keep track of multiple persons successfully. In [49], visual data is used by a robot to determine its path based on a complex decision making algorithm. This research focused on soccer player robots. Obviously the location of the ball must be tracked. Similarly, [57] uses visual data to predict the location of a soccer ball. In [33], the authors use 
SLAM (simultaneous localization and mapping) algorithm in order to determine the surrounding environment.

Underwater robotics have incorporated visual tracking systems as the other types of robotics [14]. In one approach, computer vision system is used in a ROV to track a fish [61]. Similarly, this system is used to track jelly-fish with an ROV.

\subsection{Objectives of Shark Tracking Research}

Specific objectives for the overall project were defined as:

Shark Motion Behavior Characterization This part of the research focuses on modeling the different behavior modes of a shark including, resting, foraging, etc., as well as modeling the transitions between these modes. In every mode, shark locomotion kinematics will be modeled as well as associated model certainty. Associating these modes with different oceanographic parameters and geolocated positions is also of interest.

AUV Marine Tracking Technology To track a tagged shark with a mobile platform, investigation into novel shark localization is required. Robust and intelligent planning and control in response received acoustic transmissions must be developed. This planning should consider speed, distance, and the depth of the AUV relative to the shark as well as shark movement behaviors.

\subsection{Objectives of the current research}

This thesis only focuses on "AUV Marine Tracking Technology" whose purpose is to design an autonomous mobile shark tracking system. All components of the system will be discussed in the next chapter. The validation and experimental 
results are covered in the later chapters. 


\section{Chapter 2}

\section{Mobile Tracking System Design}

\section{Problem Statement}

This thesis proposes solutions to two related problems, 1) Design of a mobile shark tracking system, and 2) Design of an autonomous control system that drives the mobile unit to the shark.

\section{$2.1 \quad$ System Design}

\subsubsection{Design Constraints}

Several constraints were considered in this design problem, including physical requirements of both the mobile technology and the shark itself. The constraints include: 1) Minimum speed, 2) Minimum maneuverability, 3) Minimum detection range, 4) Minimum sampling frequency, and 5) Full autonomy. 


\subsubsection{Average Sharks Speed}

For the mobile system to follow an individual shark, it must have a maximum speed equal to or greater than the shark's maximum sustainable swimming speed. In "Aspects of Shark Swimming Performance Determined using a Large Water Tunnel" [15], the critical speeds of several sharks were tested. Lemon, Leopard, and Mako sharks were tested for the purpose of estimating their speed. The measured speed was based on the maximum sustainable swimming speed for a set period of time, in this case 30 minutes. The type of shark which this project focuses on is Leopard sharks. In [15], a total of 18 different Leopard sharks were tested with total lengths ranging from $0.35 \mathrm{~m}$ to $1.21 \mathrm{~m}$. The average critical swimming speed was $0.70 \mathrm{~m} / \mathrm{s}$ with a maximum recorded sustainable swimming speed of $0.97 \mathrm{~m} / \mathrm{s}$ [56]. This value is used as a minimum max speed for the mobile tracking system.

\subsubsection{Sharks Maneuverability}

Another important issue to address in this work is the shark's maneuverability, or high rotational velocity. A study at University of South Florida has shown that sharks can change their directions while moving at full speed. The key to this ability is that sharks use their tiniest traits-flexible scales on their bodies. These scales control water flow separation across their bodies. Based on the experimental measurements and models of shark scales; the tapered shape of a shark's fin enables the scales to be easily manipulated to angles of 60 degrees or more [31]. This also can be rephrased in a mathematical form. In Figure 2.1, assume that the shark is going with the maximum swim speed and the vehicle is following its path. Suddenly the shark turns around and goes to the opposite 


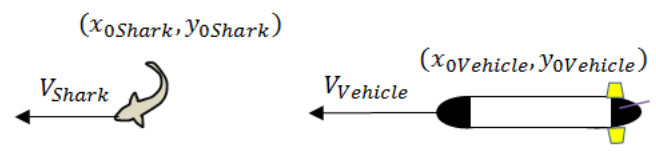

(a)

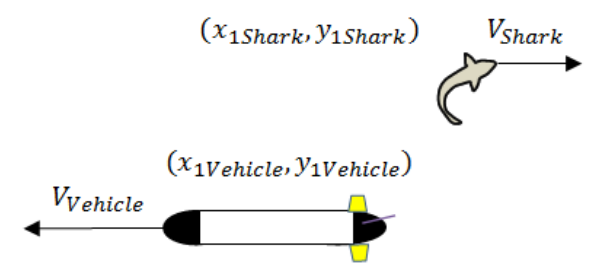

(b)
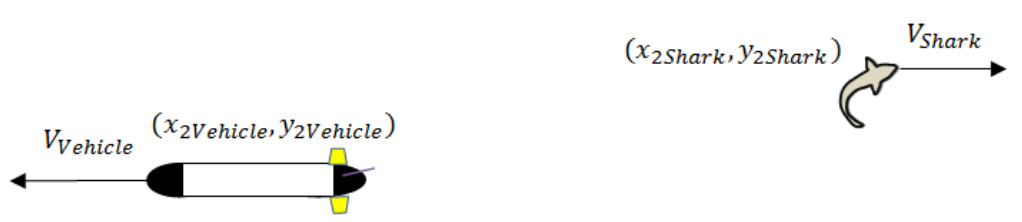

(c)
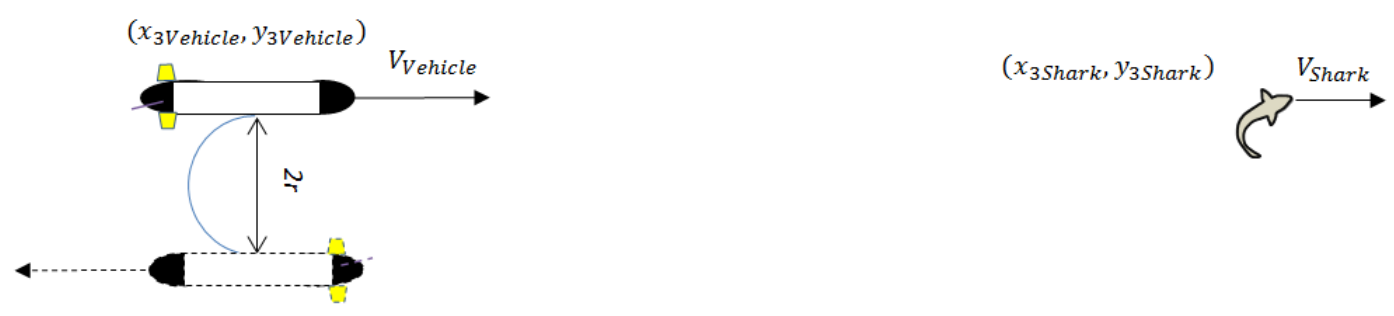

(d)

Figure 2.1: Vehicle Maneuverability Analysis 
direction.

Therefore, the vehicle detects this change after a while and it must turn around to follow the shark. Figure 2.1a presents the initial state of the vehicle and the shark. In Figure 2.1b the shark suddenly changes its direction to the opposite direction. After a while, the vehicle detects the new location of the shark (Figure 2.1c). Finally the vehicle changes its direction toward the shark and follows the shark (Figure 2.1d). To calculate the minimum acceptable rotational rate for the vehicle in this scenario, it is required to consider some factors:

$$
\Delta T_{Z}=\frac{V_{\text {Vehicle }}}{\sqrt{\left(x_{2 \text { Vehicle }}-x_{1 \text { Vehicle }}\right)^{2}+\left(y_{2 \text { Vehicle }}-y_{1 \text { Vehicle }}\right)^{2}}}
$$

Where,

$\left(x_{\text {Vehicle }}, y_{\text {Vehicle }}\right):$ The location of the tracking system.

$\left(x_{\text {Shark }}, y_{\text {Shark }}\right)$ : The location of the shark.

$V_{\text {Vehicle: }}$ Velocity of the vehicle.

$\Delta T_{Z}$ : The time between sensor measurements of the tracking device after the shark changes it direction.

When the tracking device locates the new position of the shark, the tracking vehicle turns around to follow the new direction. Assume that this turn takes $\Delta T_{T}$ seconds with the radius $\mathrm{r}$. Therefore,

$$
V_{\text {Vehicle }}=r \omega=r \frac{\Delta \theta}{\Delta t}=r \frac{\pi}{\Delta T_{T}}
$$

Where, 
$\omega$ : Angular velocity of the vehicle.

$\Delta \theta$ : Angular displacement.

While the vehicle is changing its direction, the shark moves further away. The distance that the shark goes can be calculated as:

$$
\sqrt{\left(x_{3 \text { Shark }}-x_{1 \text { Shark }}\right)^{2}+\left(y_{3 \text { Shark }}-y_{1 \text { Shark }}\right)^{2}}=\frac{V_{\text {Shark }}}{\Delta T_{T}+\Delta T_{Z}}
$$

Assume that the shark just moves directly on the x-axis. Therefore, $y_{3 \text { Shark }}=$ $y_{1 \text { Shark }}$. Also $x_{1 \text { Shark }}$ was calculated when the tracking device located the shark in the previous step. Therefore,

$$
x_{3 \text { Shark }}=\frac{V_{\text {Shark }}}{\Delta T_{T}+\Delta T_{Z}}+x_{1 \text { Shark }}
$$

The maximum distance between the shark and the vehicle must be less than the tracking device's sensing range. Therefore,

$$
\sqrt{\left(x_{3 \text { Vehicle }}-x_{3 \text { Shark }}\right)^{2}+\left(y_{3 \text { Vehicle }}-y_{1 \text { Shark }}\right)^{2}}<D R
$$

Where,

DR: Tracking device maximum detection range.

Based on the assumption, $\left(y_{3 \text { Vehicle }}-y_{1 \text { Shark }}\right)^{2}$ holds a constant value which is called A. Therefore,

$$
\left(x_{3 \text { Vehicle }}-x_{3 \text { Shark }}\right)^{2}<D R^{2}+A
$$

Therefore,

$$
\left|x_{3 \text { Vehicle }}-\frac{V_{\text {Shark }}}{\Delta T_{T}+\Delta T_{Z}}-x_{1 \text { Shark }}\right|<\sqrt{D R^{2}+A}
$$




$$
\begin{aligned}
& \left|x_{3 \text { Vehicle }}-\frac{V_{\text {Shark }}}{r_{x} \frac{\pi}{V_{\text {Vehicle }}}+\Delta T_{Z}}-x_{1 \text { Shark }}\right|<\sqrt{D R^{2}+A} \\
& \left|x_{3 \text { Vehicle }}-\frac{V_{\text {Shark }} V_{\text {Vehicle }}}{r_{x} \pi+\Delta T_{Z} V_{\text {Vehicle }}}-x_{1 \text { Shark }}\right|<\sqrt{D R^{2}+A} \\
& \left|\left(r_{x} \pi+\Delta T_{Z} V_{\text {Vehicle }}\right)\left(x_{3 \text { Vehicle }}-x_{1 \text { Shark }}\right)-V_{\text {Shark }} V_{\text {Vehicle }}\right| \\
& <\left(r_{x} \pi+\Delta T_{Z} V_{\text {Vehicle }}\right) \sqrt{D R^{2}+A} \\
& \left(r_{x} \pi+\Delta T_{Z} V_{\text {Vehicle }}\right)\left(x_{3 \text { Vehicle }}-x_{1 \text { Shark }}\right)-V_{\text {Shark }} V_{\text {Vehicle }} \\
& <\left(r_{x} \pi+\Delta T_{Z} V_{\text {Vehicle }}\right) \sqrt{D R^{2}+A} \\
& r_{x}<\frac{V_{\text {Shark }} V_{\text {Vehicle }}+\Delta T_{Z} V_{\text {Vehicle }}\left(\sqrt{D R^{2}+A}-\left(x_{3 \text { Vehicle }}-x_{1 \text { Shark }}\right)\right)}{\pi\left(x_{3 \text { Vehicle }}-x_{1 \text { Shark }}-\sqrt{D R^{2}+A}\right)} \\
& \left(r_{x} \pi+\Delta T_{Z} V_{\text {Vehicle }}\right)\left(x_{3 \text { Vehicle }}-x_{1 \text { Shark }}\right)-V_{\text {Shark }} V_{\text {Vehicle }} \\
& >-\left(r_{x} \pi+\Delta T_{Z} V_{\text {Vehicle }}\right) \sqrt{D R^{2}+A} \\
& r_{x}>\frac{V_{\text {Shark }} V_{\text {Vehicle }}+\Delta T_{Z} V_{\text {Vehicle }}\left(-\sqrt{D R^{2}+A}-\left(x_{3 \text { Vehicle }}-x_{1 \text { Shark }}\right)\right)}{\pi\left(x_{3 \text { Vehicle }}-x_{1 \text { Shark }}+\sqrt{D R^{2}+A}\right)}
\end{aligned}
$$

Therefore the minimum rotational rate for the vehicle can be calculated by Equation 2.14 if the shark moves along the $\mathrm{x}$-axis.

Now assume that the shark just moves directly on the y-axis. Therefore, $x_{3 \text { Shark }}=x_{1 \text { Shark }}$. Also $y_{1 \text { Shark }}$ was calculated when the tracking device located the shark in the previous step. Therefore,

$$
y_{3 \text { Shark }}=\frac{V_{\text {Shark }}}{\Delta T_{T}+\Delta T_{Z}}+y_{1 \text { Shark }}
$$


The maximum distance between the shark and the vehicle must be less than the tracking device's sensing range. Therefore,

$$
\sqrt{\left(x_{3 \text { Vehicle }}-x_{3 \text { Shark }}\right)^{2}+\left(y_{3 \text { Vehicle }}-y_{1 \text { Shark }}\right)^{2}}<D R
$$

Where,

DR: Tracking device maximum detection range.

Based on the assumption, $\left(x_{3 \text { Vehicle }}-x_{1 \text { Shark }}\right)^{2}$ gets a constant value which is called B. Therefore,

$$
\left(y_{3 \text { Vehicle }}-y_{3 \text { Shark }}\right)^{2}<D R^{2}+B
$$

Therefore,

$$
\begin{gathered}
\left|y_{3 \text { Vehicle }}-\frac{V_{\text {Shark }}}{\Delta T_{T}+\Delta T_{Z}}-y_{1 \text { Shark }}\right|<\sqrt{D R^{2}+B} \\
\left|y_{3 \text { Vehicle }}-\frac{V_{\text {Shark }}}{r_{y} \frac{\pi}{V_{\text {Vehicle }}}+\Delta T_{Z}}-y_{1 \text { Shark }}\right|<\sqrt{D R^{2}+B} \\
\left|y_{3 \text { Vehicle }}-\frac{V_{\text {Shark }} V_{\text {Vehicle }}}{r_{y} \pi+\Delta T_{Z} V_{\text {Vehicle }}}-y_{1 \text { Shark }}\right|<\sqrt{D R^{2}+B} \\
\left|\left(r_{y} \pi+\Delta T_{Z} V_{\text {Vehicle }}\right)\left(y_{3 V \text { ehicle }}-y_{1 \text { Shark }}\right)-V_{\text {Shark }} V_{\text {Vehicle }}\right| \\
<\left(r_{y} \pi+\Delta T_{Z} V_{\text {Vehicle }}\right) \sqrt{D R^{2}+B} \\
\left(r_{y} \pi+\Delta T_{Z} V_{\text {Vehicle }}\right)\left(y_{3 V e h i c l e}-y_{1 \text { Shark }}\right)-V_{\text {Shark }} V_{\text {Vehicle }} \\
<\left(r_{y} \pi+\Delta T_{Z} V_{\text {Vehicle }}\right) \sqrt{D R^{2}+B} \\
r_{y}<\frac{V_{\text {Shark }} V_{\text {Vehicle }}+\Delta T_{Z} V_{\text {Vehicle }}\left(\sqrt{D R^{2}+B}-\left(y_{3 V e h i c l e}-y_{1 S h a r k}\right)\right)}{\pi\left(y_{3 V e h i c l e}-y_{1 \text { Shark }}-\sqrt{D R^{2}+B}\right)}
\end{gathered}
$$




$$
\begin{gathered}
\left(r_{y} \pi+\Delta T_{Z} V_{\text {Vehicle }}\right)\left(y_{3 \text { Vehicle }}-y_{1 \text { Shark }}\right)-V_{\text {Shark }} V_{\text {Vehicle }} \\
>-\left(r_{y} \pi+\Delta T_{Z} V_{\text {Vehicle }}\right) \sqrt{D R^{2}+B} \\
r_{y}>\frac{V_{\text {Shark }} V_{\text {Vehicle }}+\Delta T_{Z} V_{\text {Vehicle }}\left(-\sqrt{D R^{2}+B}-\left(y_{3 \text { Vehicle }}-y_{1 \text { Shark }}\right)\right)}{\pi\left(y_{3 \text { Vehicle }}-y_{1 \text { Shark }}+\sqrt{D R^{2}+B}\right)}
\end{gathered}
$$

Therefore the minimum rotational rate for the vehicle can be calculated by Equation 2.25 if the shark moves along the y-axis.

Finally the minimum rotational rate for the vehicle to perform the operation can be calculated by Equation 2.26 as follows:

$$
r>\sqrt{r_{x}^{2}+r_{y}^{2}}
$$

\subsubsection{Sharks' Sensing Range}

Sharks are very sensitive animals. They can pick up on electromagnetic fields, sounds, smells, tastes, and physical activities. Therefore, one issue is to design the tracking vehicle in a way that sharks do not attack it. Also, it must maintain a minimum distance from the shark to reduce the probable damages. Shovelnose and Leopard sharks inhabit shallower waters and tend to swim on the bottom. They have the tendency to rest on the bottom and mill around over an area of 100 meters. Therefore, the vehicle must be able to stay at least 20 meters away from these sharks. Current research focuses on Leopard sharks which are not as sensitive to local disturbances. Therefore this issue is not considered in the current research. 


\subsubsection{Necessity of Autonomy}

To relieve human operators of the tedious, difficult and sometimes dangerous task of continuous shark tracking experiments that can last up to 48 hours, this thesis aims to design a system that is fully autonomous with no human intervention except to start and stop the system.

\subsubsection{Tracking Device Response Time}

As discussed in the previous sections; sharks can change their speed and direction with their maximum speed. This can happen any time during operations and the tracking system must be able to detect the changes as fast as they happen. For this purpose, the tracking device must get measurements through its sensors

on regular frequency $\frac{1}{\Delta T_{Z}}$ which discussed in the previous sections. Also, the tracking system must be able to log the state of the shark in regular time steps during operations.

\subsubsection{Design Criteria}

\subsubsection{Tracking Speed}

Based on the average swim speed of sharks, the tracking vehicle must be able to operate even faster than the average speed of shark. As discussed before, the greatest sustainable velocity of Leopard sharks is $0.97 \mathrm{~m} / \mathrm{s}$. If the shark is far away from the tracking system, the vehicle must be able to approach the shark with a greater speed to reduce the distance in between. 


\subsubsection{System Maneuverability}

The tracking system must also be able to change its direction as fast as possible. As discussed before, sharks can change their direction with their full speed. Therefore, two common scenarios might happen: 1) The shark and the vehicle are close to each other when the shark turns in a random direction, and 2) They are far enough from each other. The main problem is the first case. In the first case, the vehicle must be able to turn very fast, which requires a greater moment of inertia to the rudder and greater thrusting force (with respect to the vehicle size). This would help the vehicle to turn with a smaller radius to follow the shark.

\subsubsection{Tracking Device Response Time and Detection Range}

To perform consistent shark tracking operations, the tracking device must be able to localize the shark on a regular frequency. The higher frequency of logging data is more desirable. This is because by having more information about the shark's movements, localization of the shark can be done easier which increases the reacting time of the tracking vehicle. Also, the more detecting range is more desirable. Therefore, having a tracking device with higher frequency and greater detection range can be defined as a constraint for the system.

\subsubsection{Duration of System in Hours}

In terms of migratory habits, sharks can be divided into three groups; 1) local sharks, 2) coastal pelagic sharks and 3) highly pelagic sharks [28].

- Local Sharks: are those that do not migrate at all. They stay within 
approximately 160 kilometers (or 100 miles) of a central point [28].

- Pelagic Sharks: will migrate along the shallower waters for a distance of over 1600 kilometers, or 1000 miles. This may be to follow certain currents or in search of food [28].

- Highly Pelagic Sharks: which will follow currents across oceanic borders $[28]$.

Having a system which can operate consistently for a longer time would help to collect more information about the migration paths. Therefore a system that can follow sharks on longer migratory paths is desirable. Table 2.1 summarizes the constraints and criteria to design a tracking system.

\begin{tabular}{|c|c|c|}
\hline & Design Constraints & Design Criteria \\
\hline Speed & $\begin{array}{l}\text { Leopard Sharks Maximum } \\
\text { Speed is: } \\
V_{\text {Shark }}=0.97 \mathrm{~m} / \mathrm{s}\end{array}$ & $\begin{array}{l}\text { The maximum speed of the } \\
\text { vehicle must be greater than } \\
\text { sharks' maximum speed. }\end{array}$ \\
\hline Maneuverability & $\begin{array}{l}\text { Sharks can change their } \\
\text { direction with maximum } \\
\text { speed. }\end{array}$ & $\begin{array}{l}\text { The vehicle must be able to } \\
\text { maintain a great angular ve- } \\
\text { locity to be able to follow } \\
\text { the shark consistently. }\end{array}$ \\
\hline Sensing Range & $\begin{array}{l}\text { Sharks can smell up to } 100 \\
\text { meters away but they can- } \\
\text { not see or hear anything far- } \\
\text { ther than } 30 \text { meters. }\end{array}$ & $\begin{array}{l}\text { At least } 30 \text { meters away } \\
\text { from the shark. (Not appli- } \\
\text { cable to this thesis) }\end{array}$ \\
\hline $\begin{array}{l}\text { Response Time and } \\
\text { Detection Range }\end{array}$ & $\begin{array}{l}\text { As fast as shark moves } \\
\text { within a specific range. }\end{array}$ & $\begin{array}{l}\text { The tracking device must } \\
\text { be able to detect the shark } \\
\text { with a greater maximum de- } \\
\text { tection range and report the } \\
\text { location of shark with con- } \\
\text { stant sampling frequency. }\end{array}$ \\
\hline
\end{tabular}

Table 2.1: Tracking System Design Constraints and Criteria 


\subsection{Proposed Solution}

To design an autonomous system for shark tracking, three components must be used: 1) A vehicle for tracking, 2) A tracking device, and 3) A shark. The vehicles for tracking may vary in type, e.g., it can be an autonomous underwater vehicle (AUV), a remotely operated vehicle (ROV) or even a boat which human track a shark manually. Also, the tracking device may vary in type, e.g., an acoustic transmission device, a GPS device or a visual tracking system. The candidate vehicles and the tracking devices have advantages and disadvantages in comparison with one another which are discussed below.

\subsubsection{Vehicle for Tracking}

As discussed the first chapter, one of the goals of designing autonomous systems is to reduce operations labor. Therefore, it is required to have a robot to perform the operation. There are two choices to select a robot which would perform shark tracking operation. A remotely operated vehicle (ROV) is a type of an underwater robot which is operated via the support unit by a human. The ROV is connected to the support unit by a long tether which limits the range of the robot. An important part of our shark tracking project was to design a system which can follow a shark as long as possible without any human intervention, making ROVs less applicable. The other choice was to select an AUV to perform the operation. AUVs can operate without human control and they do not have any wire or tether which limit their functionality. Therefore, an AUV was chosen as the tracking vehicle. 


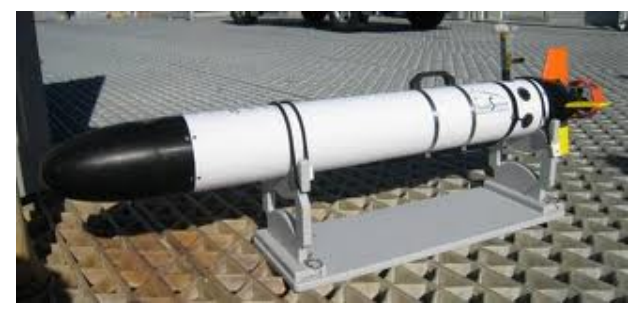

Figure 2.2: An AUV

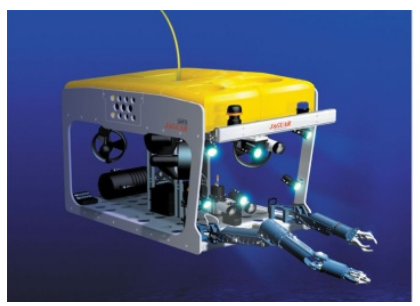

Figure 2.3: A ROV

\subsubsection{Device for Tracking}

The chosen vehicle (AUV) must use a tracking system to provide measurements necessary for shark localization. Three main types of tracking were considered: 1) Acoustic tracking systems device, 2) GPS tracking systems, and 3) Visual tracking systems $[22,30,23,56]$.

\subsubsection{Acoustic Tracking Device}

An acoustic tracking device uses the transmission and reception of sound waves to localize its target's locations. Often, an acoustic transmitter tag is attached to the target being tracked. The receiving systems consist of an array of hydrophones which can be processed for calculating relative distances and or bearings to the tag. To note, acoustic devices perform well in deep waters.

Previous research focused on using acoustic tracking system for tracking targets and positioning systems for autonomous underwater vehicle. Acoustic tracking systems are widely used for tracking of fishes $[22,30,23,56]$. In [20], researchers tagged a Macrofauna and established an array of hydrophones in the Mullica River and Great Bay estuary in southern New Jersey, USA as part of an observatory for the study of migration. In [18], the authors used an AUV to track two tagged Atlantic Sturgeon in the Hudson Rive using acoustic tracking 
system and tracked and followed the fish with a detection range of about $2 \mathrm{~km}$. [35] focused on tracking Megamouth sharks using acoustic an tracking system. In that research, the authors had followed a Megamouth shark for about 4 days. The detection range of the acoustic system in this papers was reported 200 meters. In another study, Humpback whales were tracking using acoustic tracking system. In this paper, two high density clusters of detections occurred approximately 1500 m northeast of the Hawaiian Islands and were attributed to a large aggregation of migrating animals [36].

Acoustic systems are also used for the purpose of localizing AUVs when they are underwater and have no access to GPS signals. There are three common types of acoustic positioning systems: 1) Ultrashort baseline (USBL), 2) Short baseline (SBL), and 3) Long baseline (LBL). The distance between acoustic baselines is generally used to define an acoustic positioning system which is the distance between the active sensing elements [52]. USBL was used for AUV navigation system in [42]. In this paper, the USBL sensor was attached to a boat. The USBL system interacted with the AUV frequently. To evaluate the accuracy of the USBL acoustic tracking system, AUV was driven in a square shaped area and the error was calculated by having the actual location and the estimated location of the AUV. The average error in this paper is reported as less than 20 meters. In [48] a SBL system is incorporated for an AUV navigation. In this paper, the SBL system includes 3 hydrophones that were placed on the vertices of a triangle which constantly receive signals from the transmitter. To investigate the accuracy of the system, an experiment in a 200 meters x 200 meters area was done which showed less than 10 meters average error. [34] addresses the design of a low-cost LBL navigation system for an AUV. In this paper, the experiments were done in a 500 meters $\mathrm{x} 500$ meters area in order to navigate the AUV in a 
lawnmower pattern. The average error is reported as 5 meters which presents a good accuracy.

In [13], an acoustic tracking system consisting of an array of hydrophones was used to locate the location of an acoustic tag by an AUV. In the experiments the average error reported as less than 50 meters. While there are different types of acoustic tracking system, there are a few types of portable systems which can be integrated with the vehicle. Theses systems can repeatedly locate the individuals without additional handling and transmit signals through fresh and salt water. On the other hand, portable acoustic tracking systems have a good range $(<1000 m)$ capability [53]. Any obstacle, depth, and salinity reduces the signal rate. Noise and air reduce signal detection and it requires in-water detection system (hydrophone) which increases the wet area of the AUV which increases the frictional force [8].

\subsubsection{GPS Tracking Device}

A GPS tracking device is a device that uses the Global Positioning System to determine the precise location of a vehicle, person, or other asset to which it is attached and to record the position of the asset at regular intervals. The nominal

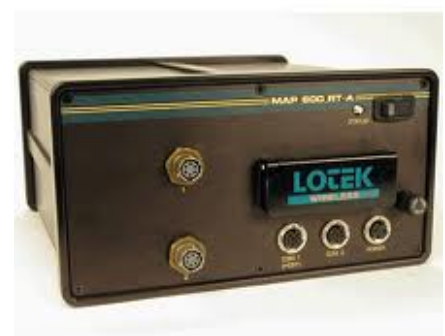

Figure 2.4: An Acoustic Tracking Device 
GPS Operational Constellation consists of 24 satellites that orbit the earth in 12 hours. There are often more than 24 operational satellites as new ones are launched to replace older satellites. The satellite orbits repeat almost the same ground track (as the earth turns beneath them) once each day. The orbit altitude is such that the satellites repeat the same track and configuration over any point approximately each 24 hours ( 4 minutes earlier each day). There are six orbital planes (with nominally four SVs in each), equally spaced (60 degrees apart), and inclined at about fifty-five degrees with respect to the equatorial plane. This constellation provides the user with between five and eight SVs visible from any point on the earth. Civil users worldwide use the Standard Positioning Service (SPS) without charge or restrictions. Most receivers are capable of receiving and using the SPS signal. The SPS accuracy is intentionally degraded by the DOD by the use of Selective Availability. SPS predictable accuracy is 10 meter on average on land while GPS signals cannot penetrate water at all [58, 7, 8]. GPS device records the location data frequently. The recorded location data can be stored within the tracking unit, or it may be transmitted to a central location data base, or internet-connected computer, radio, or satellite modem embedded in the unit. This allows the asset's location to be displayed against a map backdrop either in real time or when analyzing the track later, using GPS tracking software. Advantages of GPS tracking system can be listed as follows: 1) High spatial accuracy and temporal resolutions, 2) Capacity to collect large databases about environments, 3) Ability to localize without human aid, and 4) Ability to locating any individuals. Considering the advantages, GPS tracking systems have an important disadvantage that makes it not suitable for the tracking project.

The disadvantage of GPS is that signals cannot be received below the water's surface and most fish and sharks swim at depth. Due to this restriction, Pop-up 


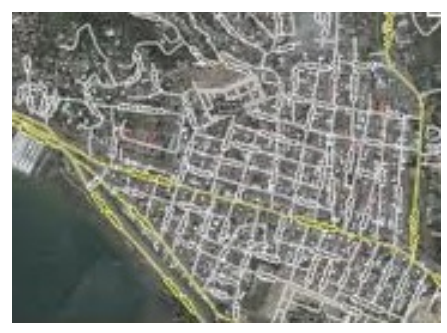

Figure 2.5: An Example of a Satellite Image

Satellite Archival Tags (PSAT) are widely used instead [29]. A PSAT is generally constructed of several components: a data-logging section, a release section, a float, and an antenna. The release sections includes a energetically popped off release section or a pin that is actively corroded on a preset date or after a specified period of time. Some disadvantages of using PSAT are their depth limitations (2000m), size, their costs, and their vulnerability to loss by environmental issues (biofouling) or ingestion by a predator [29]. For data collection the tag must be recovered which would be hard [8].

\subsubsection{Visual Tracking Systems}

Visual tracking is the process of locating a moving object (or multiple objects) over time using a camera. It has a variety of uses, some of which are: humancomputer interaction, security and surveillance, video communication and compression, augmented reality, traffic control, medical imaging and video editing. Previously, vision systems have been used for AUV navigation and pipe tracking $[59,3]$ with acceptable error rate. However, the error rate increases when the object is far away from the camera or acquired data is distorted due to camera movements. Computer vision system is inexpensive to use, but can be a time consuming process due to the number of data that is contained in video [59]. Adding further to the complexity is the possible need to use object recognition 


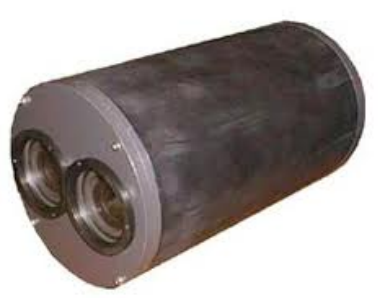

\section{Figure 2.6: An Underwater Video Camera Used by Visual Tracking Devices}

techniques for tracking. To note, the water clarity is a major issues when using vision systems in underwater environments, making it less applicable to shark tracking.

\subsubsection{System Design Decision}

The procedure to make the final decision to design the autonomous tracking system is represented in Figure 2.7. As the specifications of tracking vehicles and tracking device were discussed, a combination of an AUV and an acoustic transmission system was chosen.

\subsubsection{Final Design Decision}

Table 2.2 compares several tracking systems by constraints and criteria which discussed earlier. There are several capital letters in each criterion and constraint.

- 3 stands for good which means that the variable is in or more than the required range.

- 2 stands for moderate which means that the variable is close the required range.

- 1 stands for bad which means that the variable is not in the required range. 


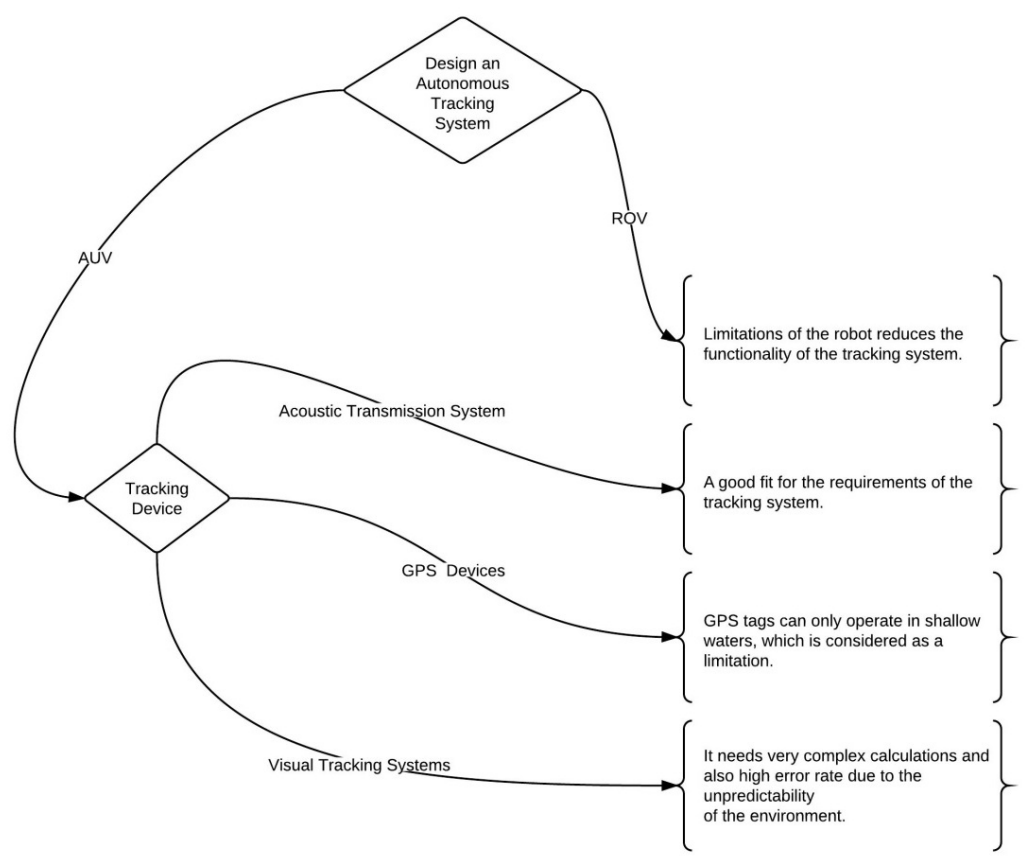

Figure 2.7: The Autonomous Tracking System Design Decision Diagram

Finally, with the total score of $24 / 24$, the autonomous tracking system was designed based on an autonomous underwater vehicle equipped with an acoustic tracking system.

\subsubsection{Current Research AUV}

IVER2, made by the ocean-server Company, was used in this research. Specifications of this AUV are presented in the Table 2.3.

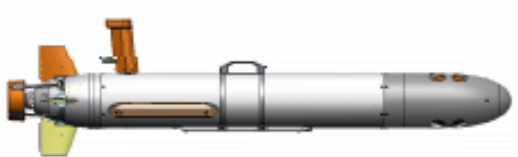

Figure 2.8: Autonomous Underwater Vehicle Used in the Current Research 


\begin{tabular}{|c|c|c|c|c|c|}
\hline $\begin{array}{l}\text { Tracking } \\
\text { Vehicle }\end{array}$ & $\begin{array}{l}\text { Tracking De- } \\
\text { vice }\end{array}$ & Constraints & & Criteria & \\
\hline \multirow[t]{5}{*}{ AUV } & Acoustic & Speed & 3 & Speed & 3 \\
\hline & Tracking & Maneuverability & 3 & Maneuverability & 3 \\
\hline & System & Sensing Range & 3 & Sensing Range & 3 \\
\hline & & Response Time & 3 & Response Time & 3 \\
\hline & & Total: & 12 & & 12 \\
\hline \multirow[t]{5}{*}{ AUV } & GPS Tracking & Speed & 3 & Speed & 3 \\
\hline & System & Maneuverability & 3 & Maneuverability & 3 \\
\hline & & Sensing Range & 2 & Sensing Range & 1 \\
\hline & & Response Time & 2 & Response Time & 1 \\
\hline & & Total: & 10 & & 8 \\
\hline \multirow[t]{5}{*}{ AUV } & Visual Track- & Speed & 3 & Speed & 3 \\
\hline & ing System & Maneuverability & 3 & Maneuverability & 3 \\
\hline & & Sensing Range & 1 & Sensing Range & 1 \\
\hline & & Response Time & 1 & Response Time & 1 \\
\hline & & Total: & 8 & & 8 \\
\hline \multirow[t]{5}{*}{ ROV } & Acoustic & Speed & 1 & Speed & 1 \\
\hline & Tracking & Maneuverability & 3 & Maneuverability & 2 \\
\hline & System & Sensing Range & 3 & Sensing Range & 3 \\
\hline & & Response Time & 3 & Response Time & 3 \\
\hline & & Total: & 10 & & 9 \\
\hline \multirow[t]{5}{*}{ ROV } & GPS Tracking & Speed & 2 & Speed & 1 \\
\hline & System & Maneuverability & 3 & Maneuverability & 2 \\
\hline & & Sensing Range & 2 & Sensing Range & 1 \\
\hline & & Response Time & 2 & Response Time & 1 \\
\hline & & Total: & 9 & & 5 \\
\hline \multirow[t]{5}{*}{ ROV } & Visual Track- & Speed & 2 & Speed & 1 \\
\hline & ing System & Maneuverability & 3 & Maneuverability & 2 \\
\hline & & Sensing Range & 1 & Sensing Range & 1 \\
\hline & & Response Time & 1 & Response Time & 1 \\
\hline & & Total: & 7 & & 5 \\
\hline
\end{tabular}

Table 2.2: Final Design Decision Table

The AUV (Figure 2.8) operates with two processors. The main processor runs the controlling API for the basic controlling operations such as fins controller, propeller velocity controller, GPS data logger, altimeter data logger, gyroscope data logger, and battery data logger. The secondary processor runs the controller 


\begin{tabular}{ll}
\hline Hull Diameter: & $0.147 \mathrm{~m}(5.8 \mathrm{in})$ \\
Hull Material: & Carbon Fiber Tube \\
Vehicle Length: & $1.27 \mathrm{~m}(50 \mathrm{in})$ \\
Weight in air: & $19 \mathrm{~kg}(42 \mathrm{lbs})$. \\
Trim Weight: & $1.2 \mathrm{~kg}(2.6 \mathrm{lbs})$. \\
Maximum Operating Depth: & $100 \mathrm{~m}(328 \mathrm{ft})$. \\
Energy: & $600 \mathrm{watt} /$ hours \\
Propulsion: & DC brushless motor \\
Control: & 4 independent fin control \\
& surfaces, yaw, pitch and ac- \\
& tive roll correction \\
Integrated Sensors: & Depth (pressure), Altimeter \\
& (acoustic), 3 Axis Digital \\
& Compass, leak detectors \\
Processor: & Standard processor is an \\
& AMD LX800. Custom \\
& CPU, Intel ATOM 1.6Ghz \\
\hline
\end{tabular}

Table 2.3: The Autonomous Underwater Vehicle Specifications

code that has been developed by the author to operate autonomously to track the shark. For the tracking purposes, this AUV has not been equipped with any tracking devices. To perform the acoustic tracking, a LOTEK acoustic system was integrated into the AUV and connected to the secondary processor via port RS-232. Together the acoustic receiver and its software supply the required information to perform the localization. The information provided by the acoustic receiver and the data from the main processor allow the controller program to localize and track the shark. The information flow block diagram is presented in Figure 2.9. In this figure, the acoustic system's hydrophones receive analog signals from the acoustic tag. MAPHost RTA interprets the information and provides the strength of the signal and bearing to the target for the secondary processor. At this stage, the main processor sends the data from GPS, gyroscope, and the power units of the main processor to the secondary processor. Using measurements from these sensors, the particle filter estimates the shark's 


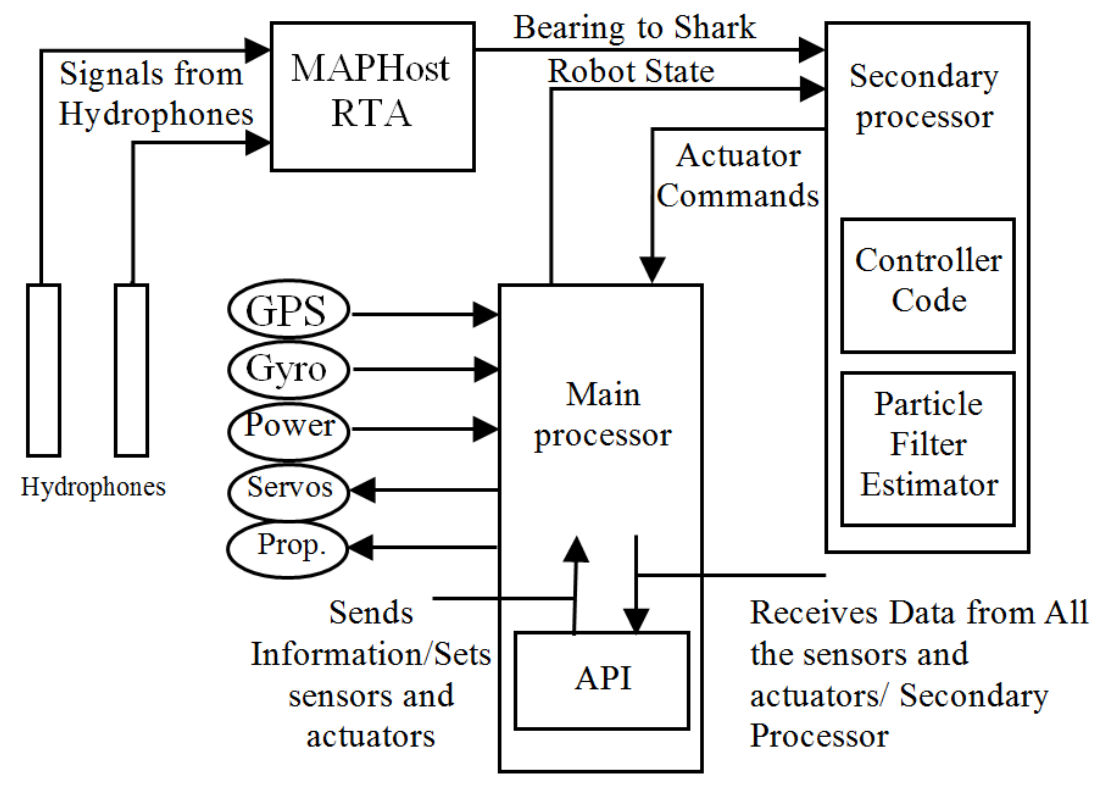

\section{Figure 2.9: System Diagram of the AUV}

location. The particle filter algorithm was not developed in this thesis. This part has been done by another member of the shark tracking team [12]. The controller code sends the proper fin angle and motor speed to the main processor allowing the AUV to track the shark at the correct distance and position.

\subsubsection{Acoustic Tracking System, LOTEK}

The LOTEK MAPHOST-RTA, Figure 2.10, is a dual acoustic receiver that has been used for the localization purposes. This device receives signals, from an acoustic transmitter which is attached to the shark, by two passive hydrophones which listen to the pings during an operation. The main usage of this device is for outdoor purposes such as boats and piers. The LOTEK system calculates the angle to the transmitter by triangulation between two hydrophones and the transmitter. 


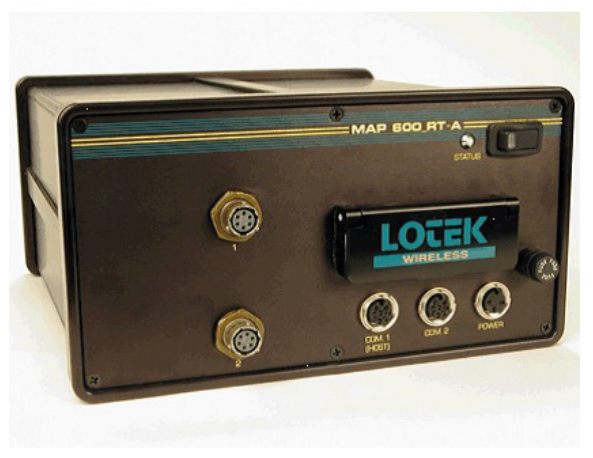

\section{Figure 2.10: Acoustic Tracking Device, LOTEK MAPHOST-RTA}

The major problem of the LOTEK system is that the bearing to the tag is always positive while this assumption is not always true. Consider an imaginary line between two hydrophones, from the perspective of the LOTEK system there is no difference between the left side $(0$ to $\pi)$ and the right side $(0$ to $+\pi)$ of the line. More details will be discussed in the controller system section.

The acoustic transmitter operates in $76 \mathrm{kHz}$ frequency range and this offers a theoretical maximum range of 500-1000 meters depending on outside noises, placement of hydrophones, and temperature differences. The acoustic transmitter can also record the depth of operation. An advantage is that the LOTEK system converts the analog signal to the digital by itself this allows the system to use thousands of tags in the same frequency. Each tag's unique ID is coded within the signal, allowing identification of each individual tag. Therefore, multiple objects can be followed by an AUV with the same frequency tags. The receiver calculates the bearing to each tag (with respect to their IDs) and provide it to the control unit. 


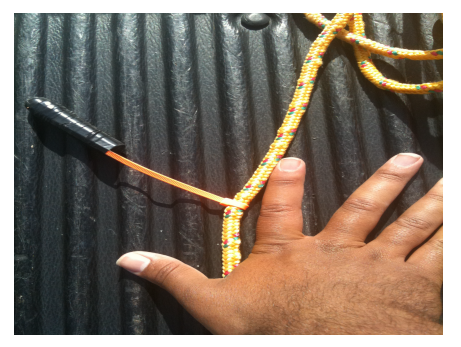

Figure 2.11: Acoustic Transmitter

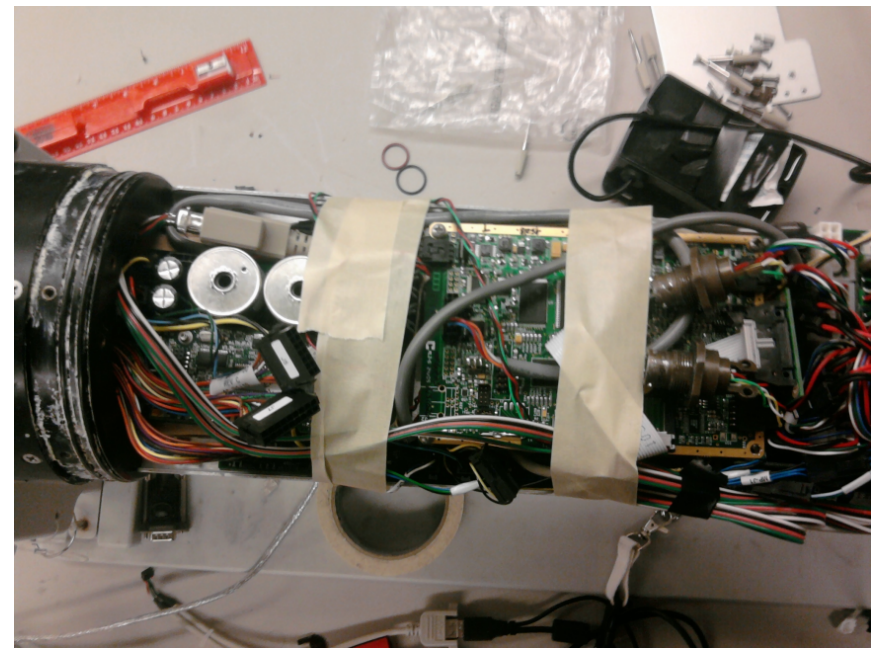

Figure 2.12: Acoustic Tracking Device Integrated with the AUV 


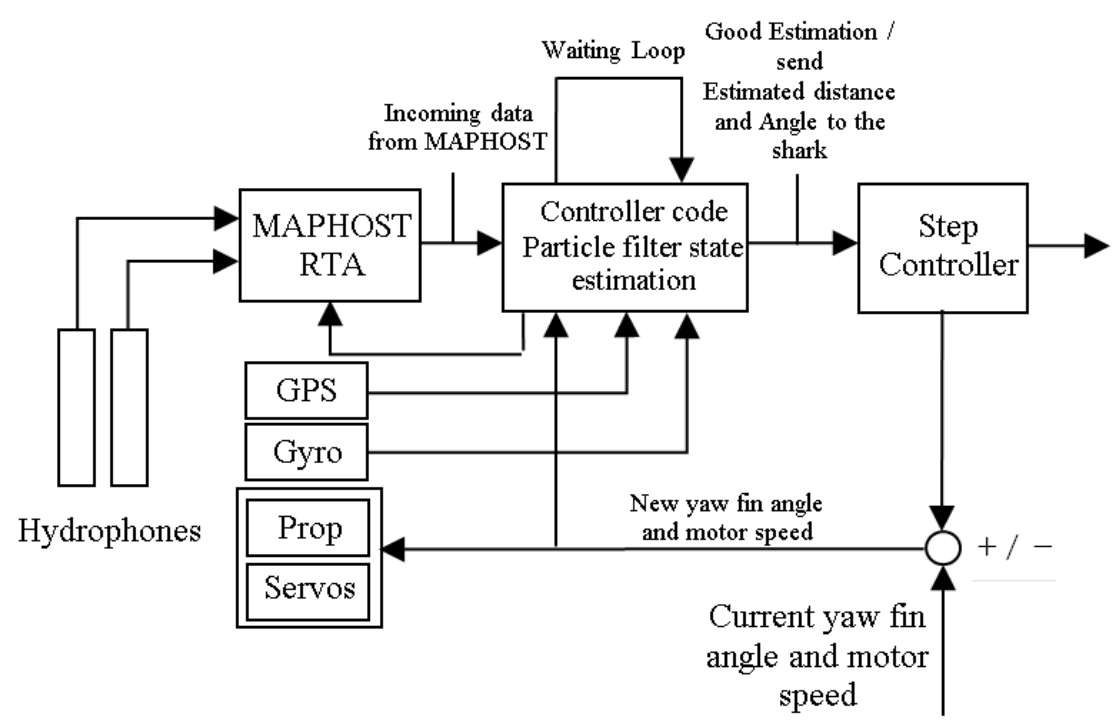

Figure 2.13: Control System Diagram of the AUV

\subsubsection{Shark Tracker System Components}

Figure 2.13 illustrates the feedback control system designed for tracking and following purposes. In each time step, the acoustic receiver waits for the signal's arrival from the acoustic tag. After a signal is received, the signal is filtered and transformed to a bearing and signal strength measurement that can be used by the state estimator. Meanwhile, the particle filter algorithm uses; the current location of the AUV from the GPS, the heading angle from the gyroscope, and the incoming data from LOTEK to update its shark state estimate. Whenever the particle filter estimates the location of the shark, it sends the absolute location of the shark with the AUV to the step controller. The step controller calculates the current error and sets the proper fin angles and propeller motor velocity. 


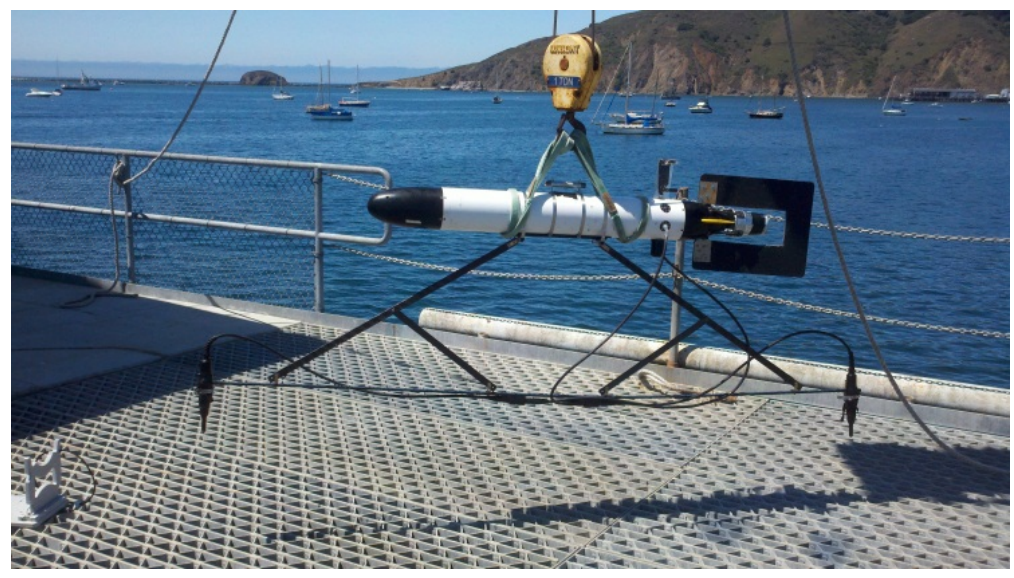

Figure 2.14: The Rig Used to Fix the Hydrophones under the AUV

\subsubsection{Hydrophone Mounting Frame}

One of the major problems encountered in the project was how to correctly attach the hydrophones to the AUV. Experiments were conducted on multiple rigs equipped with hydrophones in order to achieve admissible speed and maneuverability results. The main goals were to minimize water friction and maximize speed. The most accurate bearing calculations by the LOTEK system happen when the hydrophones are 2.4 meters from each other and 1.5 meters below the water surface. Thus several frames have been designed and tested to guarantee the maximum maneuverability and velocity. The frames were built with aluminum pipes, PVC pipes, and carbon fiber rods. After drag experiments, the final rig was built using carbon fiber rods Figure 2.14. Tables 2.4 and 2.5 present the thrusting forces needed by the AUV to move with a constant speed with each mounting frame. 


\begin{tabular}{lccc}
\hline Direction & Straight & Left Turn & Right Turn \\
\hline Carbon Fiber (N) & 9.94 & 10.131 & 9.54 \\
PVC Pipe (N) & 12.41 & 16.31 & 15.13 \\
\hline
\end{tabular}

Table 2.4: Average Thrust Force Needed for Each Mounting Frame on Forward Direction

\begin{tabular}{lccc}
\hline Direction & Straight & Left Turn & Right Turn \\
\hline Carbon Fiber (N) & 4.91 & 3.93 & 3.74 \\
PVC Pipe (N) & 7.543 & 5.88 & 4.31 \\
\hline
\end{tabular}

Table 2.5: Average Thrust Force Needed for Each Mounting Frame on Backward Direction

\subsubsection{Yaw Fin}

After completing a final design of the frame, the wet area of the AUV had been increased due to the hydrophones and carbon fiber rods. As a result, the rotational drag increased the AUV turning radius. In the case of tracking, the turning radius was too large to track the shark in a specific path. As illustrated in the Figure 2.15. IVER2 has two small yaw fins. New larger fins were built. After testing, the fin that provided the best turning radius with the minimum speed reduction was chosen (2.16). In Table 2.6, the ability to turn with the two types of fins is evaluated.

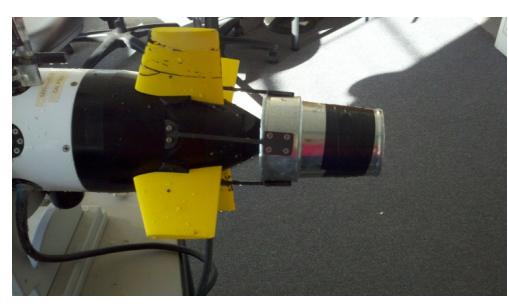

Figure 2.15: Original Yaw Fin

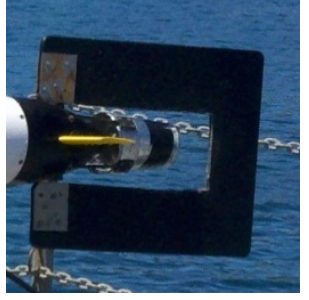

Figure 2.16: New Yaw Fin

Increasing the size of the fin would increase the control surface area leading 
to a reduction of the turning path. However if this area is increased more than a tolerable limit on the motor torques, the rotors would be burned out.

\begin{tabular}{lcccccc}
\hline Turning Angle (Degrees) & $\mathbf{1 5}$ & $\mathbf{3 0}$ & $\mathbf{4 5}$ & $\mathbf{6 0}$ & $\mathbf{7 5}$ & $\mathbf{9 0}$ \\
\hline Original Fin (Seconds) & 5.303 & 8.351 & 10.131 & 14.764 & 17.313 & 23.13 \\
New Fin (Seconds) & 4.313 & 7.543 & 9.64 & 12.011 & 15.765 & 18.095 \\
\hline
\end{tabular}

Table 2.6: Time Elapsed for the AUV to Complete a Turn with Carbon Fiber Mounting Frame and Different Yaw Fins 


\section{Chapter 3}

\section{Control System}

For this section, we assume the mobile tracking unit design will be based on Autonomous Underwater Vehicle (AUV) technology. A control system must be designed that enables the AUV to track and follow the shark using by setting the appropriate motor speed and control surface (i.e. fin) angles in response to the AUV and shark state estimates.

\subsection{Control System Design}

Figure 3.1 presents a top down view of an AUV and a shark in an environment. The AUV's state consists of current location $\left(x_{A U V}, y_{A U V}\right)$, yaw angle $\left(\theta_{A U V}\right)$, velocity $\left(V_{A U V}\right)$ and angular velocity $\left(\omega_{A U V}\right)$. The shark's state is defined by the estimated shark location $\left(x_{\text {Shark }}, y_{\text {Shark }}\right)$ and its angle $\left(\theta_{\text {Shark }}\right)$. Therefore,

$$
\widehat{X}_{(t) A U V}=\left[x_{(t) A U V} y_{(t) A U V} \theta_{(t) A U V} \dot{x}_{(t) A U V} \dot{y}_{(t) A U V} \dot{\theta}_{(t) A U V}\right]
$$




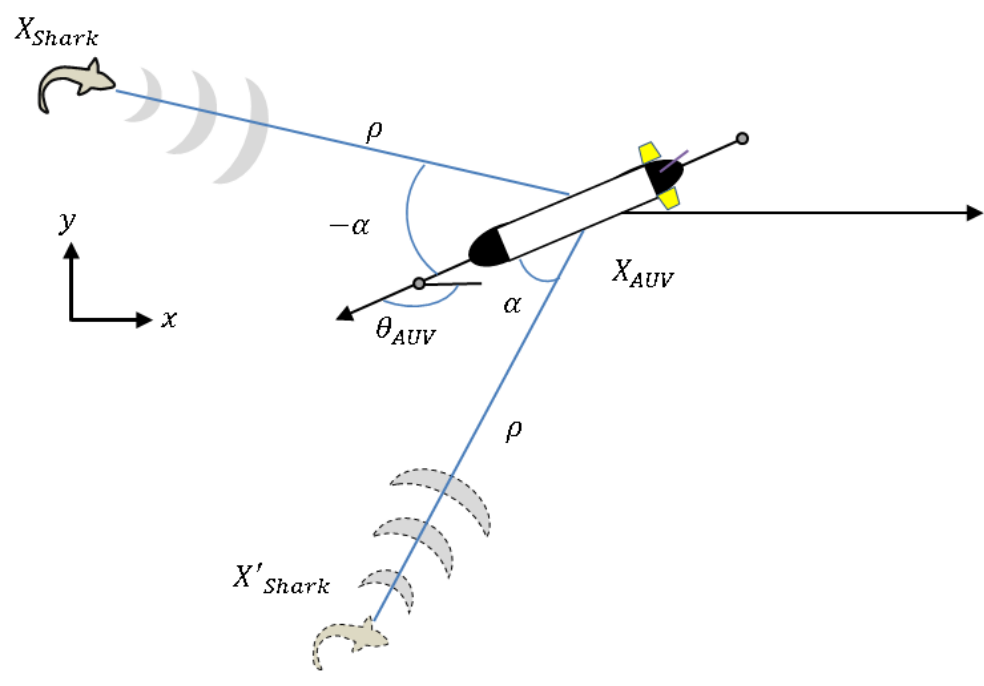

Figure 3.1: Shark Tracking Coordinate System

And,

$$
\widehat{X}_{(t) \text { Shark }}=\left[x_{(t) \text { Shark }} y_{(t) \text { Shark }} \theta_{(t) \text { Shark }}\right]
$$

Therefore distance between shark and AUV would be,

$$
\rho_{t}=\sqrt{\left(x_{(t) A U V}-x_{(t) \text { Shark }}\right)^{2}+\left(y_{(t) A U V}-y_{(t) \text { Shark }}\right)^{2}}
$$

Also the absolute bearing from the AUV to shark in the global coordinate frame is,

$$
\alpha_{t}=-\theta_{(t) A U V}+\operatorname{atan} 2\left(y_{(t) \operatorname{Shark}}-y_{(t) A U V}, x_{(t) \operatorname{Shark}}-x_{(t) A U V}\right)
$$

The reason that the coordinate system is chosen to be $2 \mathrm{D}$ is that the acoustic tag can measure the depth of the shark. Also, the final experiments were performed in very shallow waters which restricted the ability to dive. 


\subsubsection{Cost Function Definition}

The goal of the controller is to minimize the cost function defined in Equation

3.5. That is, the distance between the shark and the AUV, averaged over time.

$$
C=\frac{1}{N-t_{0}} \sum_{t=t_{0}}^{N} \rho_{t}
$$

\subsubsection{Constraints for Modeling}

The AUV has several physical constraints which must be considered. These are framed as kinematic constraints here. Dynamic constraints are ignored here and should be considered for future work.

$$
\begin{gathered}
x_{t}=x_{t-1}+\Delta t v_{t} \cos \left(\theta_{t-1}\right) \\
y_{t}=y_{t-1}+\Delta t v_{t} \sin \left(\theta_{t-1}\right) \\
\theta_{t}=\theta_{t-1}+\Delta t \omega_{t}
\end{gathered}
$$

Where,

$x_{t}$ : Position of the AUV on the $x$-axis at time $\mathrm{t}$.

$y_{t}$ : Position of the AUV on the $y$ - axis at time $\mathrm{t}$.

$\Delta t$ : The time which the AUV moved from the initial location (at time " $t-1 ")$ to the final location (at time " $t ")$.

$v_{t}$ : Velocity of the AUV (at time $t$ ). 
$\theta_{t}$ : Heading bearing of the AUV (at time $t$ ).

$\omega_{t}$ : Angular velocity of the AUV.

Equations 3.6, 3.7, and 3.8 are the kinematic equations of motion for the AUV. The maximum velocity of the AUV is 4 knots (which is 2.057 meter/second, based on conversion rate of 1 knot $=0.5144$ meters) and maximum turn radius of 3 meters [37]. Therefore the maximum angular velocity can be calculated by,

$$
\omega_{\max }=\sqrt{\frac{V_{t}}{R}}=0.83\left(\frac{\mathrm{rad}}{\mathrm{sec}}\right)
$$

Therefore the constraints can be set to:

$$
\begin{aligned}
& V_{t} \leq V_{\text {max }} \\
& \omega_{t} \leq \omega_{\text {max }}
\end{aligned}
$$

\subsubsection{Assumptions}

As discussed earlier, modeling is done by a first order kinematic model. As AUV operates underwater, due to the complexity of the equations, the effect of waves and momentum and drag forces were not considered in the kinematic equations. This reduces the calculations allowing for the tracking to be easier to implement and results to be simplified. Another assumption would consider the

environment as an open water area which doesn't contain any obstacle on the AUV's path. 


\subsection{Proposed Solution - A Bearing Modulation Controller}

The design of the AUV's control system was a critical part of this research. As discussed in chapter one, the LOTEK system waits for the signals to arrive from acoustic transmitter and then calculates relative bearing by using data from the hydrophone which receives the signal first. Also, the LOTEK system cannot differentiate between negative and positive angle values.

To improve shark localization, a control method is proposed that enables active localization, in which the AUV actively modifies its behavior to improve shark localization accuracy. To reduce localization uncertainty, the controller uses a repeated series of control actions that enable multple sensor vantage points while still guiding the AUV towards the shark, (Figure 3.2). The series consists of 3 actions which include 1) tracking a heading +45 degrees from the estimated bearing angle to the shark, 2) tracking a heading -45 degrees from the estimated bearing angle to the shark, and 3) tracking a heading equal to the estimated bearing angle to the shark. By tracking desired headings that are 90 then 45 degrees from one another, the AUV should obtain bearing measurements with sensor footprints that only overlap on one side of the vehicle. This will lead to particles on one side of the vehicle having higher weights and hence resampling will converge particles to the correct location.

Figure 3.2 provides an example. Initially, when the AUV is at point A, resampling from the Particle Filter reduces the group of particles to two clusters. After the AUV moves to point (B), the overlap of the bearing measurement sensor footprint only covers one of the particle clusters, giving those particles higher weights and high likelihood of survival during the upcoming resampling. The 
surviving particles will likely only reside in close proximity with a single (correct) cluster.

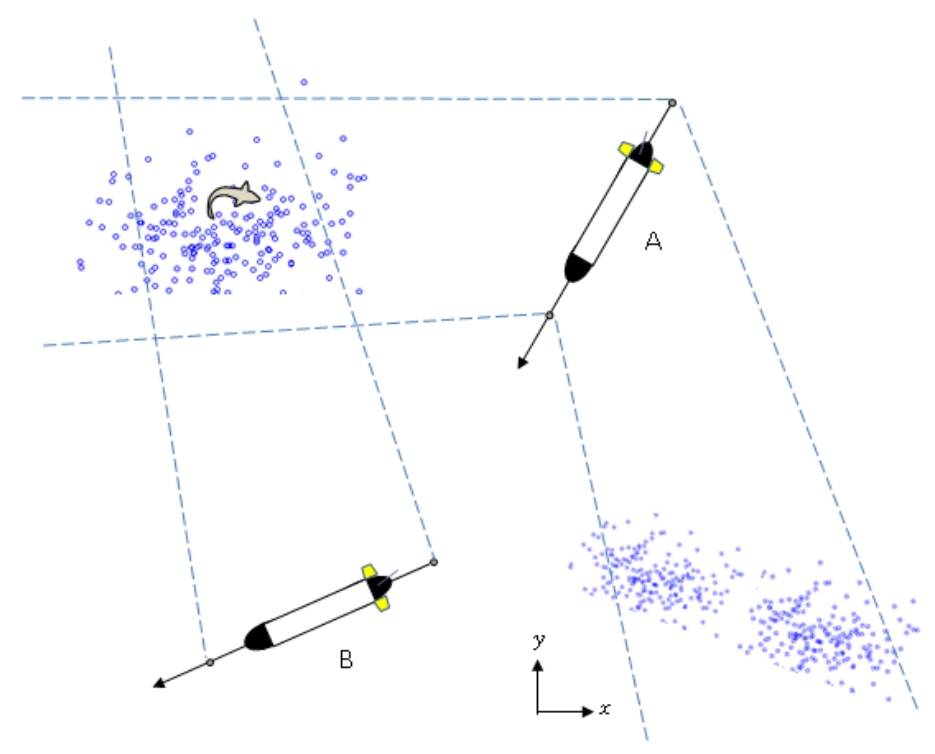

Figure 3.2: Particles Convergence

\subsubsection{Step Control System}

A proportional control system is a type of linear feedback control system, often control systems are designed using proportional control system. In the proportional control algorithm, the controller output is proportional to the error signal, which is the difference between the set point and the process variable. In other words, the output of a proportional controller is the multiplication product of the error signal and the proportional gain. This can be mathematically expressed as, 


$$
P_{\text {out }}=K_{p} e(t)
$$

Where,

$P_{\text {out }}$ : Output of the proportional controller.

$K_{p}$ : Proportional gain.

$e(t)$ : Instantaneous process error at time ' $t$ '.

To use this controller for shark tracking purpose, it is required to define output, proportional gain and the error respectively. The output can be defined as the fins' angle relative to the current bearing to the shark; the proportional gain value will be set through experiments and the error can be defined as follows:

$$
e(t)=\theta_{\text {des }}-\theta
$$

Where,

$\theta_{\text {des }}:$ Desired yaw angle in absolute coordinate frame.

$\theta$ : Vehicle yaw angle with respect to the global coordinate frame.

\subsubsection{Control System Definitions}

Let's assume,

$$
\vec{X}_{A U V}=\left[\begin{array}{ll}
x_{A U V} & y_{A U V}
\end{array}\right]
$$

And,

$$
\vec{X}_{\text {Shark }}=\left[x_{\text {Shark }} y_{\text {Shark }}\right]
$$




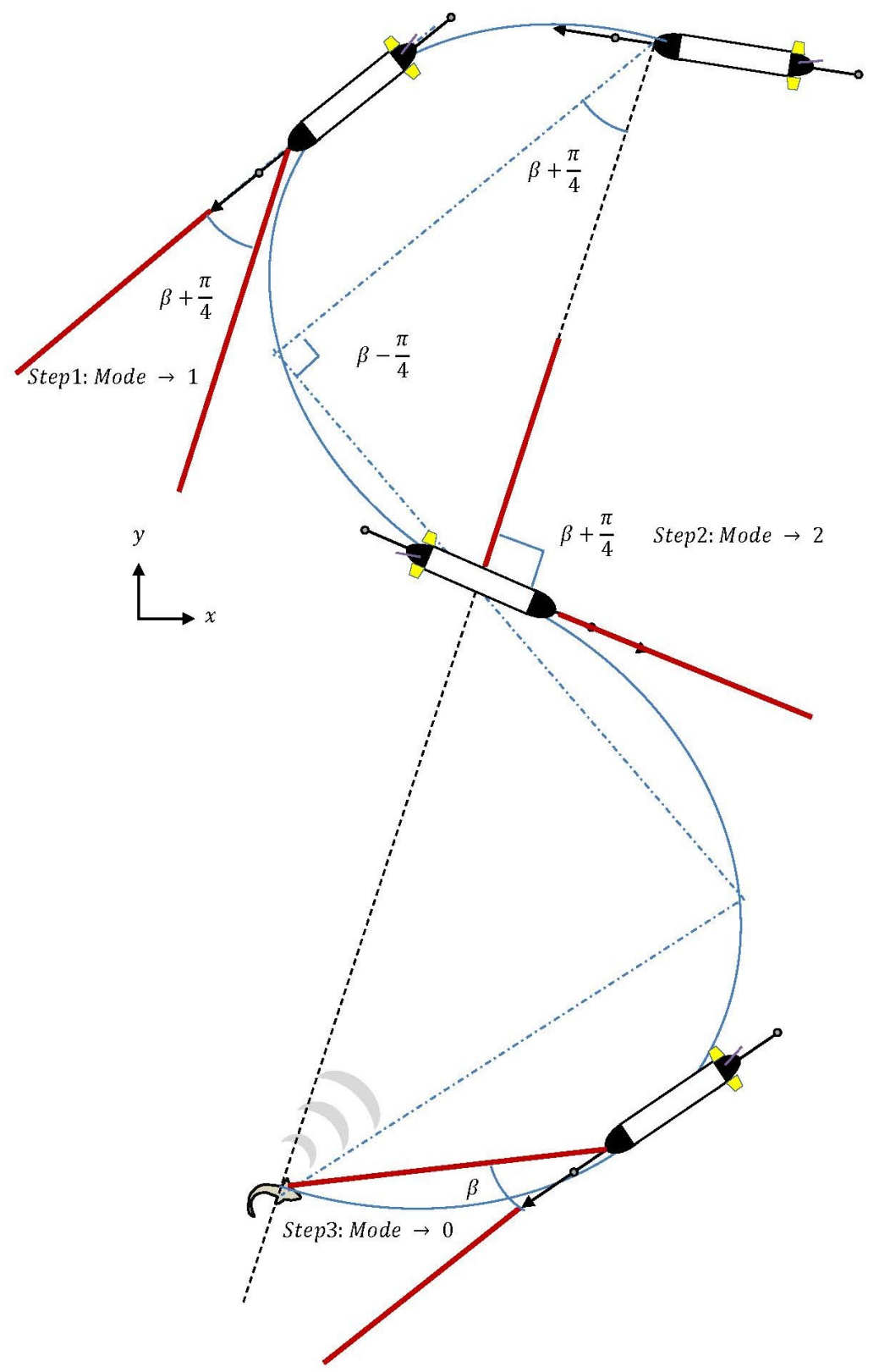

Figure 3.3: Control System Behavior 
As is known,

$$
\begin{gathered}
\rho=\left\|\vec{X}_{A U V}-\vec{X}_{\text {Shark }}\right\| \\
\rho=\sqrt{\left(x_{A U V}-x_{\text {Shark }}\right)^{2}+\left(y_{A U V}-y_{\text {Shark }}\right)^{2}}
\end{gathered}
$$

Also the absolute bearing angle from the AUV to the shark with respect to the global coordinate frame is:

$$
\beta=\operatorname{atan} 2\left(y_{\text {Shark }}-y_{A U V}, x_{\text {Shark }}-x_{A U V}\right)
$$

As defined earlier in the control system section, $\theta_{\text {des }}$ is the desired yaw angle in absolute coordinate frame. Therefore, the AUV should obey the following rules and set the heading $(\theta)$ to a proper $\theta_{d e s}$,

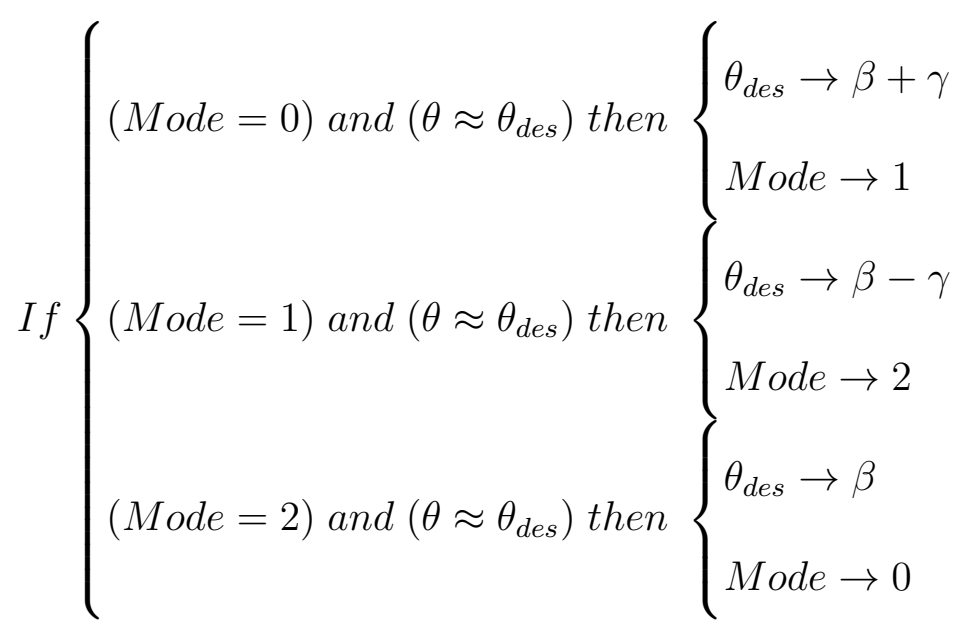

$\gamma$ : The control parameter angle (set to $\frac{\pi}{4}$ in Figure 3.3 ).

The main reason to use this $\gamma$ is to define the circularity of the path of the robot in tracking operations.

\subsubsection{Shark Tracker}

The control system receives the estimated bearing and distance to the shark from the particle filter algorithm. It sets the fins' angle and motor velocity with 
respect to those values. To relate the distance between the shark with the velocity of the AUV, Equations 3.20 and 3.21 were defined:

$$
\begin{array}{lll}
v_{t}=C_{0} V_{\max } \frac{-C_{1}}{\rho_{t}}+1 & (k n) & \rho_{t}<D \\
v=V_{\max }(k n) & \rho_{t} \geq D
\end{array}
$$

Where,

$D$ : The threshold which the AUV must lower its velocity if the distance between the AUV and the Shark is less than $D$, in the other case it must go with the maximum velocity. This variable is chosen based on experiments and Leopard sharks behavior.

Equation 3.20 sets the velocity of the AUV based on the distance between the shark and the AUV. Therefore when the AUV is getting close to the goal, its velocity is reduced and vice versa. Also, whenever the AUV is far away, more than $D$ meters from the shark, Equation. 3.21 sets the velocity of the AUV to its full speed. $V_{\max }$ was chosen to be less than the maximum velocity of the AUV because operation with the maximum propeller speed may burn the engine or wires after a long period of time.

\subsection{Simulations}

To validate the control system, the AUV, shark, and acoustic sensing system were simulated in the MATLAB environment. These simulations were used to improve the efficiency of the tracking system. The control system which was discussed earlier uses a constant value $(\gamma)$ to obtain bearing measurements with 


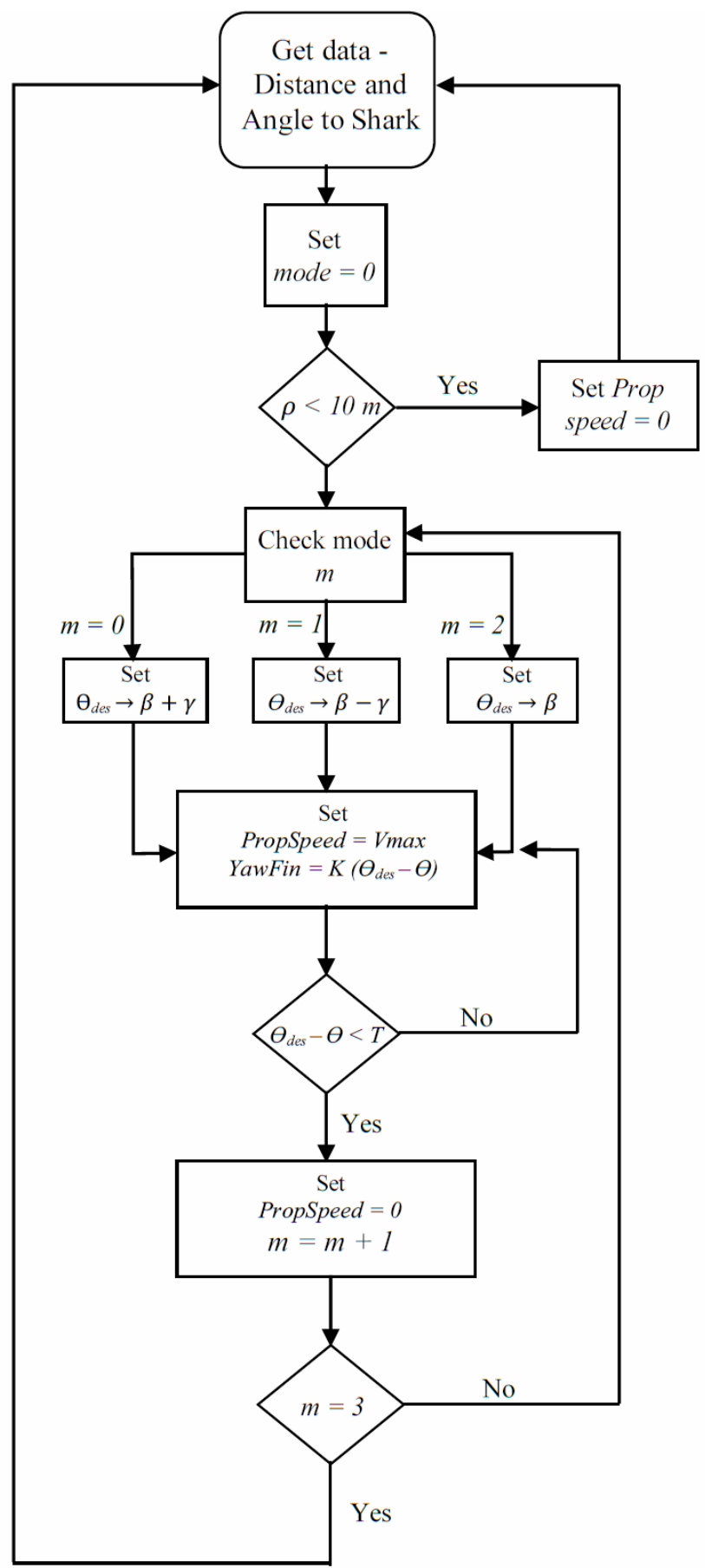

Figure 3.4: Control System Algorithm Diagram 
sensor footprints that only overlap on one side of the vehicle. Therefore by having the results of the simulations with real-time calculations, the constant value $(\gamma)$ can be dynamically set during operations.

\subsubsection{Autonomous Tracking System Simulation}

The autonomous tracking system consists of two main parts: 1) the AUV, and 2 ) the LOTEK acoustic receiver system. The simulator used to initially validate the controller was developed in MATLAB and based on models of these two main components.

\subsubsection{AUV Simulation}

The AUV is assumed to behave according to the following kinematic equations:

$$
\begin{gathered}
\widehat{X}_{A U V}=\left[x_{A U V} y_{A U V}\right] \\
\widehat{V}_{A U V}=\left[\frac{\partial x_{A U V}}{\partial t} \frac{\partial y_{A U V}}{\partial t}\right]=\left[\begin{array}{ll}
V_{x A U V} & V_{y A U V}
\end{array}\right]
\end{gathered}
$$

Where,

$$
\begin{aligned}
& \widehat{X}_{A U V} \text { : State matrix of the AUV. } \\
& \widehat{V}_{A U V} \text { : Velocity matrix of the AUV. }
\end{aligned}
$$

AUV velocity ranges from $0-4$ knots and the turning radius $(R)$ is 5 meter [37]. To calculate the rotation $(\theta)$ rate of the AUV in a small time t, one would have,

$$
d \theta_{A U V}=V_{A U V} \frac{d t}{R}
$$


By linearizing 3.24 one would have,

$$
\Delta \theta_{A U V}=V_{A U V} \frac{\Delta t}{R}
$$

After $\Delta t$ seconds, the new location of the AUV can be calculated by,

$$
\widehat{X}_{1(t) A U V}=\left[x_{0 A U V}+V_{(t) A U V} \cos \left(\Delta \theta_{A U V}\right) \Delta t y_{0 A U V}+V_{(t) A U V} \cos \left(\Delta \theta_{A U V}\right) \Delta t\right]
$$

\subsubsection{LOTEK System Simulation}

Calibration of the LOTEK system was previously done in a senior project of two undergraduate students at Cal Poly [4]. In their work, the equations were developed that map the relationship between the relative bearing and distance between the hydrophones and tag.

$$
\text { Signal }=-1 * 10^{-6} * \theta_{\text {des }}^{3}+2 * 10^{-5} * \theta_{\text {des }}^{2}+0.0947 * \theta_{\text {des }}-0.2757
$$

Where,

Signal: Value which is produced by LOTEK system..

$\theta_{\text {des }}$ : Relative bearing between the AUV and the shark.

\subsubsection{Standard Deviation}

The standard deviation of the particle position is used as a measure of confidence in the shark state estimation.

$$
\begin{aligned}
& \mu_{x}=\frac{1}{N} \sum_{i=1}^{P} x_{i} \\
& \mu_{y}=\frac{1}{N} \sum_{i=1}^{P} y_{i}
\end{aligned}
$$




$$
\begin{gathered}
\sigma_{x}^{2}=\frac{1}{N} \sum_{i=1}^{P}\left(x_{i}-\mu_{x}\right)^{2} \\
\sigma_{y}^{2}=\frac{1}{N} \sum_{i=1}^{P}\left(y_{i}-\mu_{y}\right)^{2}
\end{gathered}
$$

Where $\mu_{x}, \mu_{y}, \sigma_{x}^{2}$ and $\sigma_{y}^{2}$ are the mean positions of the particles in the $\mathrm{x}$ and $\mathrm{y}$ direction, and variance of the particles in the $\mathrm{x}$ direction and $\mathrm{y}$ direction respectively.

Assume that the calculations are done at each $\Delta t$ second. Therefore, after each time step, the new states of the simulated AUV and the simulated shark can be calculated based on their previous states. The new states help the particle filter algorithm to estimate the location of the simulated shark. On the other hand, at each time step the states of the simulated AUV, the simulated shark and the particles (in the particle clusters) can be stored. If we repeat this process for different values of $\gamma$, for a constant operation time, a database including all states with different $\gamma$ values can be produced. Thereby the database can be used in real-time calculations. So at each time step, the database is searched for the similar situations with the lowest standard deviation of the particles clusters. Finally, the $\gamma$ value is chosen from the database and applied to the controller in real-time.

\subsubsection{Simulation Data for an Improved Control System}

To produce the database, which was explained in the previous section, the simulated shark was placed in seven different locations relative to the simulated $\operatorname{AUV}(0,30,60,90,120,150,180$ degrees $)$ in a constant distance (200 m). Also for each location, six different $\gamma$ values $(0,15,30,45,60,75$ degrees) were used in 
the simulated controller for approaching the shark. In each run of the simulator, states of the simulated AUV, the simulated shark and the particles were stored at each time step. Storing the results of all states would be useful in real-time calculations. Thus, a new controller can be designed which uses this data to find the most similar situation in the database, with the lowest standard deviation, to the current situation and uses its stored $\gamma$ value to increase the convergence rate of the particles cluster. Similarity can be defined by comparing the current distance and bearing to the shark with the stored states in the database. Figure 3.5 presents examples of data generations for the database. In this set of experiments, the simulated shark is placed in 30 degrees relative to the simulated AUV. Each image presents the path that the simulated AUV traverses to follow the simulated shark with constant $\gamma$ values.

\subsubsection{Setting Controller Parameters}

Similarity between the current state of the AUV, shark and particles with the data in the database was briefly defined in the previous section. In the database six variables are stored for each time step. These variables include: 1) Relative bearing to the shark, 2) Standard deviation of the particles, 3) Heading angle of the AUV, 4) Distance to the shark, 5) Time step, and 6) $\gamma$ value. Here, the flowchart to find the optimal $\gamma$ for the controller is presented in Figure 3.6.

To investigate the results of incorporating the improved controller, a large variety of experiments were done with the constant $\gamma$ controller for different situations. The simulated shark was placed in seven locations $(0,30,60,90$, 120, 150, 180 degrees relative to the simulated AUV with 200 meters distance in between). For each position, the experiment was done with six different $\gamma$ 


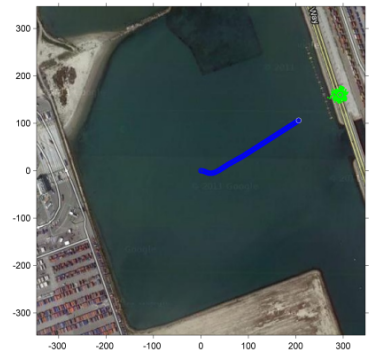

(a)

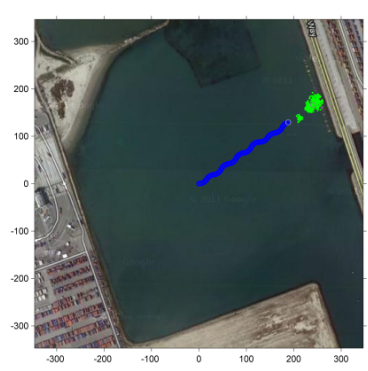

(c)

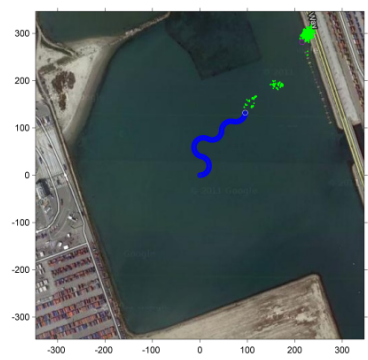

(e)

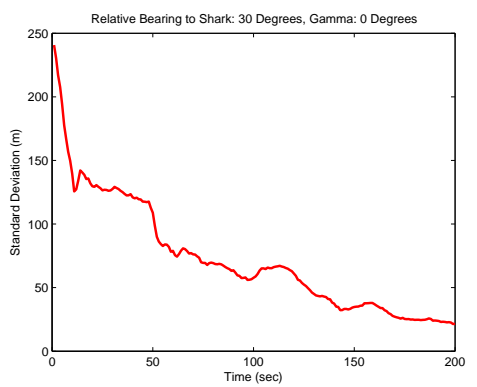

(b)

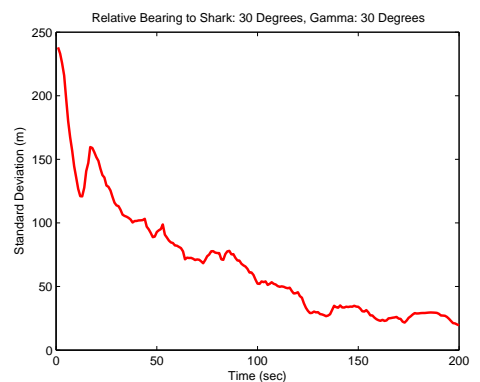

(d)

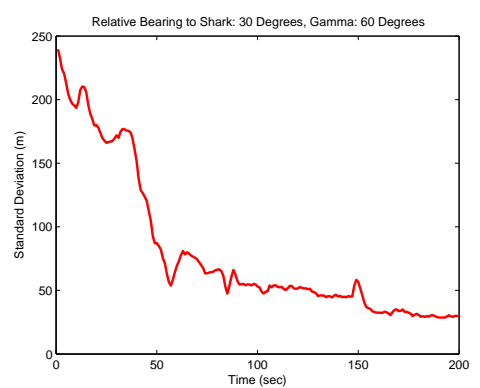

(f)

Figure 3.5: Graphs of Plotting the Particles Standard Deviation vs. Time with Different Yaw Angle Values

values $(0,15,30,45,60,75$ degrees). Then the results of experiments with the constant $\gamma$ controller with six different $\gamma$ values were averaged. On the other hand, experiments with the improved controller were done in the same situations as the constant $\gamma$ controller. Table 3.1 presents the average slopes of the first 20 seconds of running the both controllers vs. the bearing to the simulated shark. For all the bearings the average slope for the improved controller is greater. This 


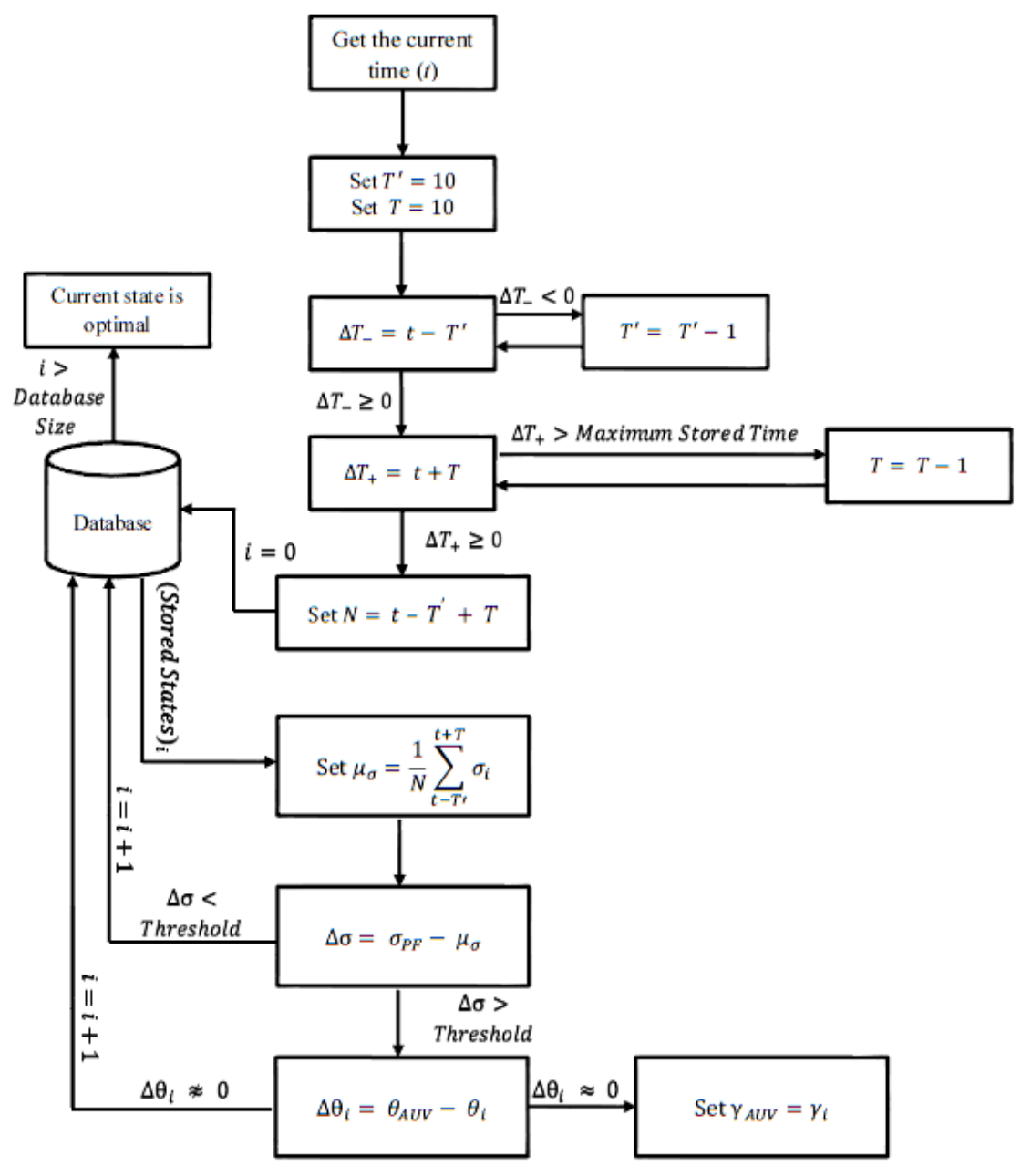

Figure 3.6: Setting Controller Parameters Algorithm

means that the particle clusters converge faster than the original controller. Table 3.1 presents the average slopes between $t=20$ to $t=200$ seconds. The results of the Table 3.2 are similar to Table 3.1 , but in two cases $\left(\frac{\pi}{6}\right.$ and $\left.\frac{\pi}{2}\right)$ the original controller decreases faster than the improved controller. This represents the fact that using the database might be enough to be to improve the controller, but it is not guaranteed that in all situations the chosen values are the optimal values. 


\begin{tabular}{lccccccc}
\hline$\theta_{\text {des }(t=0)}($ Degrees $)$ & $\mathbf{0}$ & $\frac{\pi}{6}$ & $\frac{\pi}{3}$ & $\frac{\pi}{2}$ & $\frac{2 \pi}{3}$ & $\frac{5 \pi}{6}$ & $\pi$ \\
\hline $\begin{array}{l}\text { Improved } \\
\text { Controller } \\
(\mathbf{m} / \mathbf{s})\end{array}$ & -6.286 & -7.384 & -7.0122 & -10.962 & -5.1948 & -2.6249 & -2.9432 \\
$\begin{array}{l}\text { Original } \\
\begin{array}{l}\text { Controller } \\
(\mathbf{m} / \mathbf{s})\end{array}\end{array}$ & -5.6852 & -3.898 & -3.8894 & -3.2657 & -3.561 & -1.2629 & -1.7094 \\
\hline
\end{tabular}

Table 3.1: Standard Deviation of Particles Clusters Reduction Slopes, for the first 20 seconds

\begin{tabular}{lccccccc}
\hline$\theta_{\text {des }(t=0)}($ Degrees $)$ & $\mathbf{0}$ & $\frac{\pi}{6}$ & $\frac{\pi}{3}$ & $\frac{\pi}{2}$ & $\frac{2 \pi}{3}$ & $\frac{5 \pi}{6}$ & $\pi$ \\
\hline $\begin{array}{l}\text { Improved } \\
\text { Controller } \\
(\mathbf{m} / \mathbf{s})\end{array}$ & -1.358 & -0.286 & -3.9723 & 0.703552 & -1.4208 & -4.6223 & -3.1675 \\
$\begin{array}{l}\text { Original } \\
\begin{array}{l}\text { Controller } \\
(\mathbf{m} / \mathbf{s})\end{array}\end{array}$ & -1.1221 & -1.124 & -1.3199 & -2.14236 & -0.9473 & -2.1712 & -2.0142 \\
\hline
\end{tabular}

Table 3.2: Standard Deviation Particles Clusters Reduction Slopes, after the first 20 seconds 


\section{Chapter 4}

\section{Experiments Description and Results}

To validate the system performance, a variety of ocean experiments were conducted that involved autonomous AUV tracking of either a stationary or moving tag.

\subsection{Stationary Tag Tracking}

Initial experiments were conducted at the CCMS (Cal Poly Center for Coastal Marine Science, Avila Beach, CA, shown in Figure 4.2). For stationary tag experiments, an acoustic tag was attached to an anchored rope and placed 100 meters away from the pier and 2 meters below the water's surface. The acoustic system was designed to have hydrophones 2.4 meters from each other. The acoustic tag that was used in the experiments operates with a $76 \mathrm{kHz}$ frequency and a 2 second ping rate. During these experiments, the AUV's start position relative to the tag was varied to ensure that the tracking could be performed from every direction 


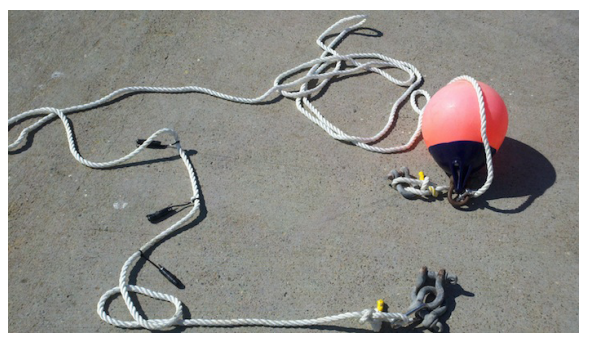

Figure 4.1: Acoustic Transmitter Attached to a Buoy

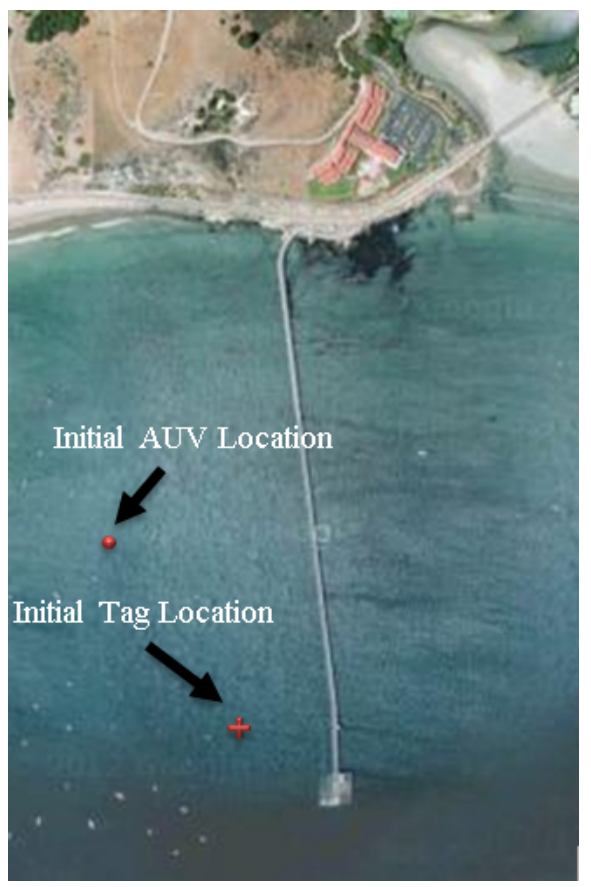

Figure 4.2: Cal Poly Pier, Avila Beach, CA.

and from various distances. In these experiments, the acoustic was attached to a rope which is connected to a buoy from a one side and an anchor from the other side. This would help to stabilize the position of the tag 2 meters below the water's surface. Figure 4.1 shows the buoy, rope, anchor and the acoustic tag that were used to stabilize the tag on a fixed location. In the Figure 4.2, a sample location of the AUV and the tag for experiments are also presented.

During the experiments, the tag was fixed on a position (e.g. (Latitude: 
35.1699, Longitude: -120.74139)). Then the AUV was placed in various positions relative to the location on the tag. A sample table of relative locations is provided in Table 4.1 Overall, close to 20 trials were done in this phase.

\begin{tabular}{cc}
\hline Tag Location $(\boldsymbol{m}, \boldsymbol{m})$ & $\boldsymbol{A} \boldsymbol{U} \boldsymbol{V}$ Start Location $(\boldsymbol{m}, \boldsymbol{m})$ \\
\hline$(0,0)$ & $(25,25)$ \\
$(0,0)$ & $(-25,-25)$ \\
$(0,0)$ & $(-25,25)$ \\
$(0,0)$ & $(25,-25)$ \\
\hline
\end{tabular}

Table 4.1: Relative Location between AUV and Tag

\subsection{Moving Tag Tracking}

After stationary tag tracking, the second set of experiments included autonomous tracking and following of a moving tag. The first set of moving tag experiments were conducted by attaching the tag to a Kayak with a two meter long rope was tied to the kayak's stern. In those experiments, the kayak traveled in a wide variation of random paths, both toward and away from the AUV. Table 4.2 presents the start locations for the both AUV and moving tag. Overall, close to 30 trials were done in this phase.

\begin{tabular}{cc}
\hline Moving Tag Start Location $(\boldsymbol{m}, \boldsymbol{m})$ & AU $\boldsymbol{V}$ Start Location $(\boldsymbol{m}, \boldsymbol{m})$ \\
\hline$(0,0)$ & $(25,25)$ \\
$(25,25)$ & $(-25,-25)$ \\
$(-25,-25)$ & $(0,25)$ \\
$(25,20)$ & $(-25,0)$ \\
\hline
\end{tabular}

Table 4.2: Relative Location between AUV and Tagged Kayak 


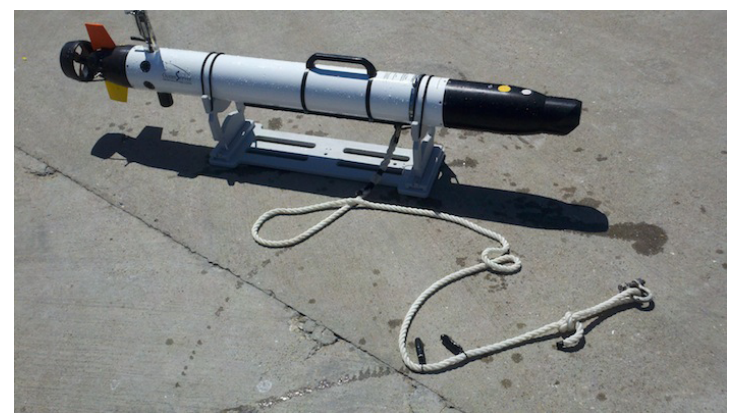

\section{Figure 4.3: Transmitter Attached to The Second AUV}

\subsection{Secondary AUV Tracking}

To provide a metric of tag state estimation accuracy, a second AUV with known position was tagged and tracked by the first AUV equipped with the tracking system. The error here is calculated as the difference between the second AUV's actual position (as measured with GPS) and the first AUV's state estimate of position as calculated by the Particle Filter that fuses bearing measurements over time.

\subsection{SeaPlane Lagoon Field Experiments}

SeaPlane Lagoon (Figure 4.4) is located in the Port of Los Angeles. The Lagoon is roughly in rectangular shape with 800 meters side. Mostly, water is shallow and many Leopard sharks can be found there at particular times of the year. This was the final location for testing and shark tracking of the season. Stationary tag and two AUV tracking tests were repeated before tracking a shark. Subsequently, a leopard shark (Triakis semifasciata) was caught, externally fitted with an acoustic transmitter tag, and released.

The last results demonstrate the accuracy and ability of the system in per- 


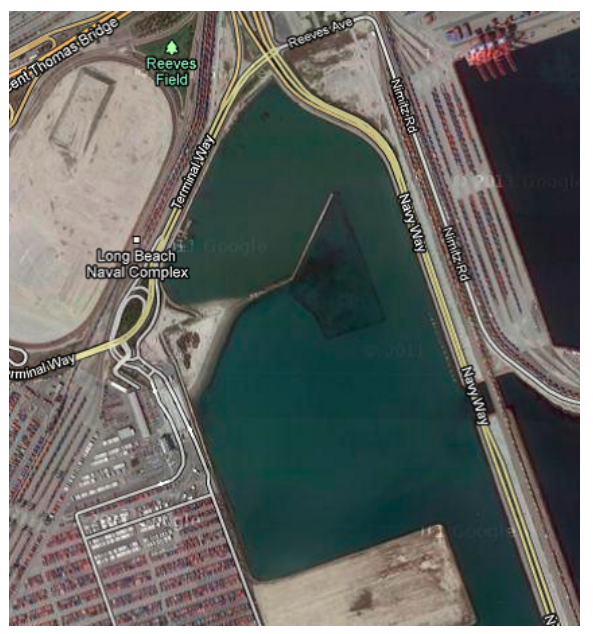

Figure 4.4: SeaPlane Lagoon, Los Angeles, CA.

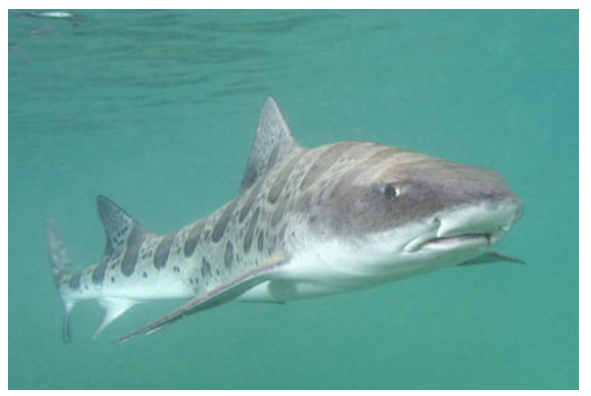

Figure 4.5: A Leopard Shark

forming tracking operations. In the Long Beach experiments, the shark was pulled to the surface using the line, and was then gently restrained with a rope tied to its tail. Sharks have a biological response of tonic immobility, becoming still as though they are hypnotized, when they are turned upside down. This was used to keep the shark from moving while it was fitted with the tag. The entire procedure took less than 10 minutes. Once the tagged shark was released, the AUV was deployed to track and follow the shark. 


\subsection{Results}

\subsubsection{Stationary Tag Tracking}

In this experiment, the tag was fixed at a specific location and the AUV starts from several different locations around the tag. At each time step, the state of the AUV is logged by the GPS device and gyroscope mounted on the AUV. Also, the particle filter algorithm estimates the location of the tag by getting measurements from the LOTEK device. Therefore, to evaluate the accuracy of the system, the particle filter algorithm estimations must be compared with the actual values.

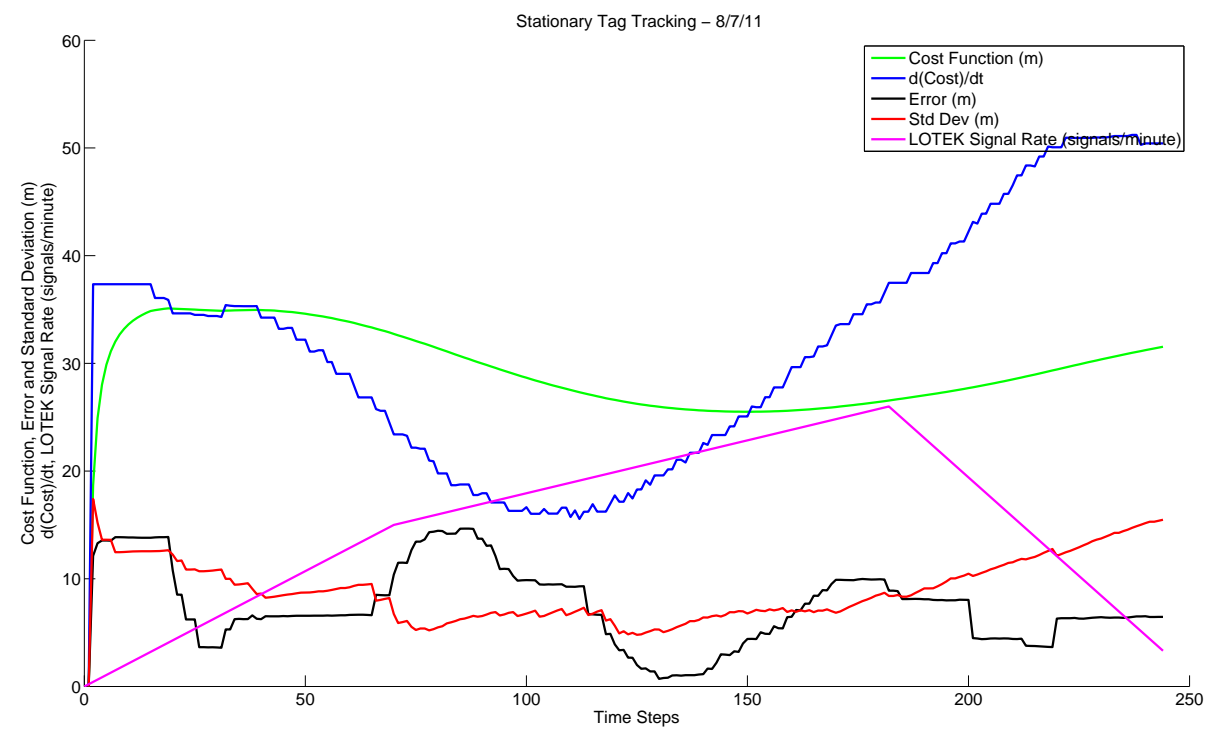

Figure 4.6: Cost Function, Error, Standard Deviation, Cost Function First Derivative, and LOTEK Signal Rate from Tracking a Stationary Tag

Figure 4.6 presents the results of an experiment which was done in 250 seconds. In Figure 4.6, the cost function is plotted in green and it increases with different slope during the experiment. To show how it changes, the first derivative of the cost function is also plotted in blue. The first derivative of the cost 
function increases at the beginning of the experiment due to lack of information about the location of the tag. Therefore, as the LOTEK signal rate increases (plotted in pink) and the AUV gets close to the localized tag, the first derivative of the cost function decreases. Also, when LOTEK signal rate increases by the time, the standard deviation and the error values decrease significantly. This means that the particle filter algorithm receives enough measurements from the LOTEK device to perform the estimation. At the end of the mission, the standard deviation increases because of signal loss. On the other hand, when the LOTEK signal rate increases by the time, cost function increases less than the time which the LOTEK signal rate decreases. Error in this experiment defined as the distance between the actual and estimated tag locations:

$$
e_{t}=\sqrt{\left(x_{T a g}-x_{E s t .}\right)^{2}+\left(y_{T a g}-y_{E s t .}\right)^{2}}
$$

\subsubsection{Tagged AUV Tracking}

For the second round of experiments; the secondary AUV (IVER2) was tagged with an acoustic transmitter. Like the stationary tag tracking, the acoustic tag was attached to the AUV and fixed 2 meter under the water's surface. This experiment was done in about an hour. In this experiment, data on the second AUV such as GPS logs and gyroscope (for heading bearing) logs were compared with the values which the follower AUV estimated about the second AUV. This comparison helps to calculate the error value.

As presented in Figure 4.7 at the beginning of the mission, the error value is lower in comparison with the second half of the mission. The high error value resulted from the area being covered with sea weed which acted as a wall against 


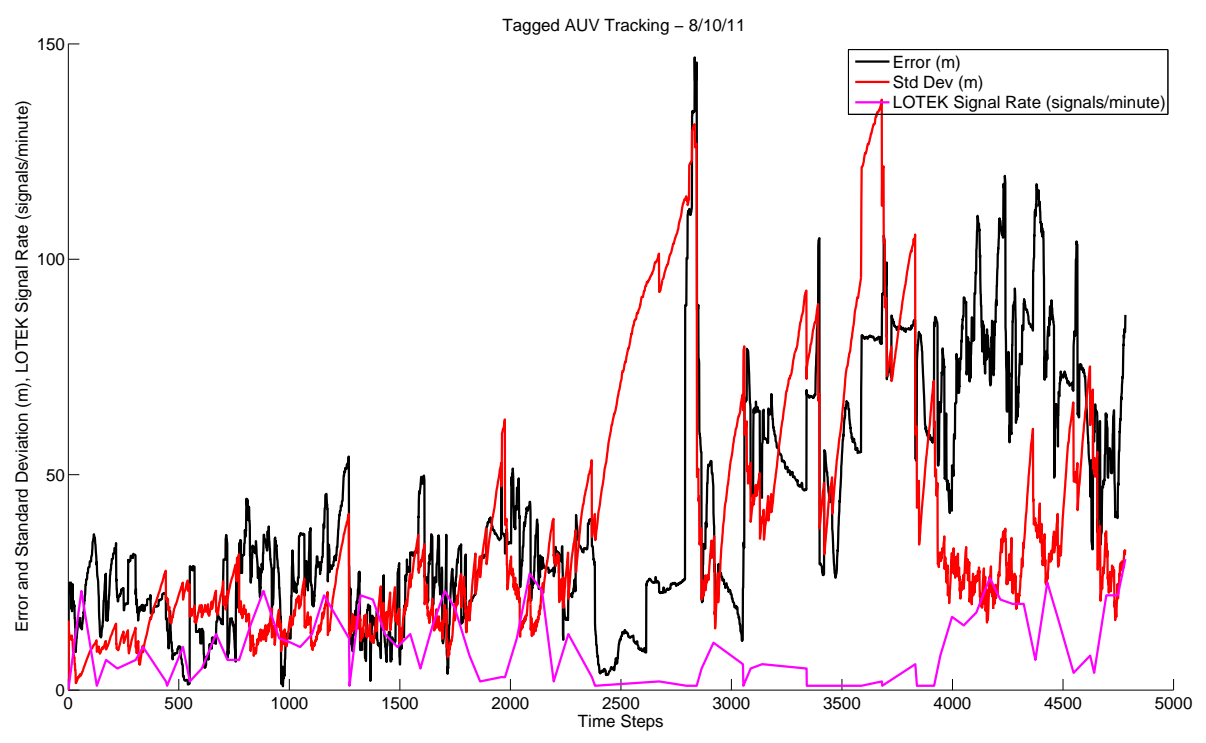

Figure 4.7: Error, Standard Deviation, and LOTEK Signal Rate from Tracking a Tagged AUV

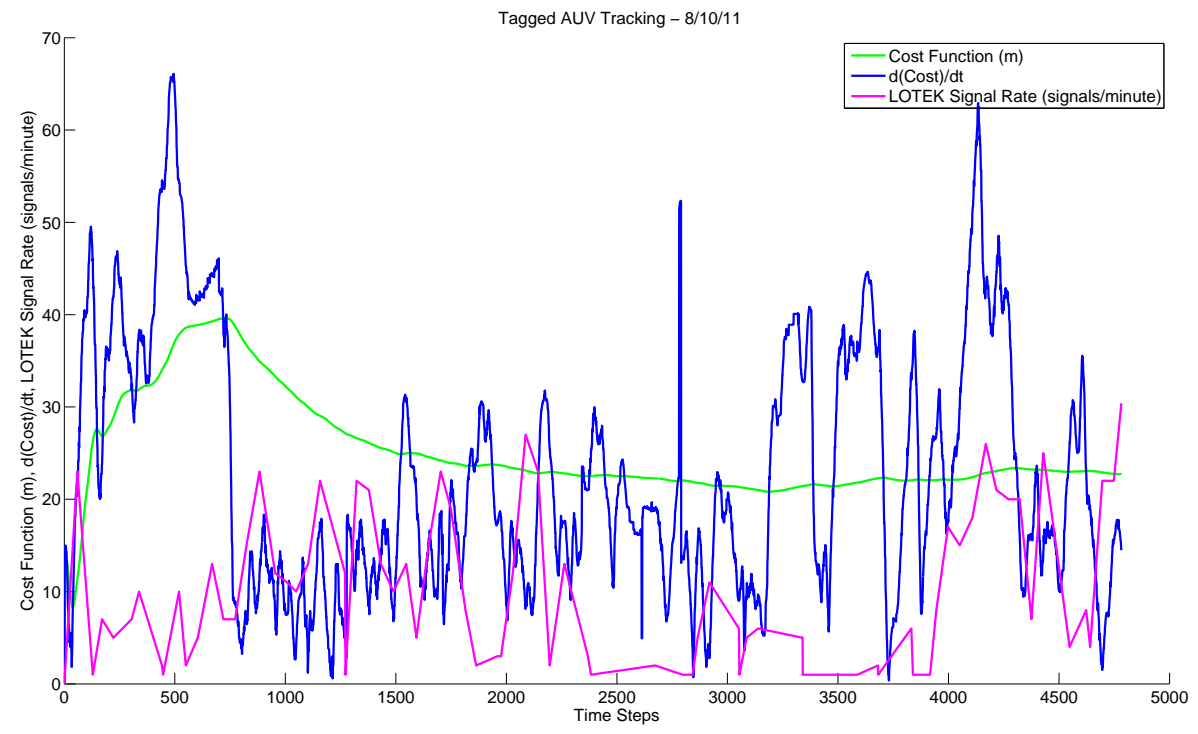

Figure 4.8: Cost Function, Cost Function First Derivative, and LOTEK Signal Rate from Tracking a Tagged AUV

acoustic signals and blocked them from the receiver. Therefore by having fewer signals, the error and standard deviation increased respectively until the AUV 


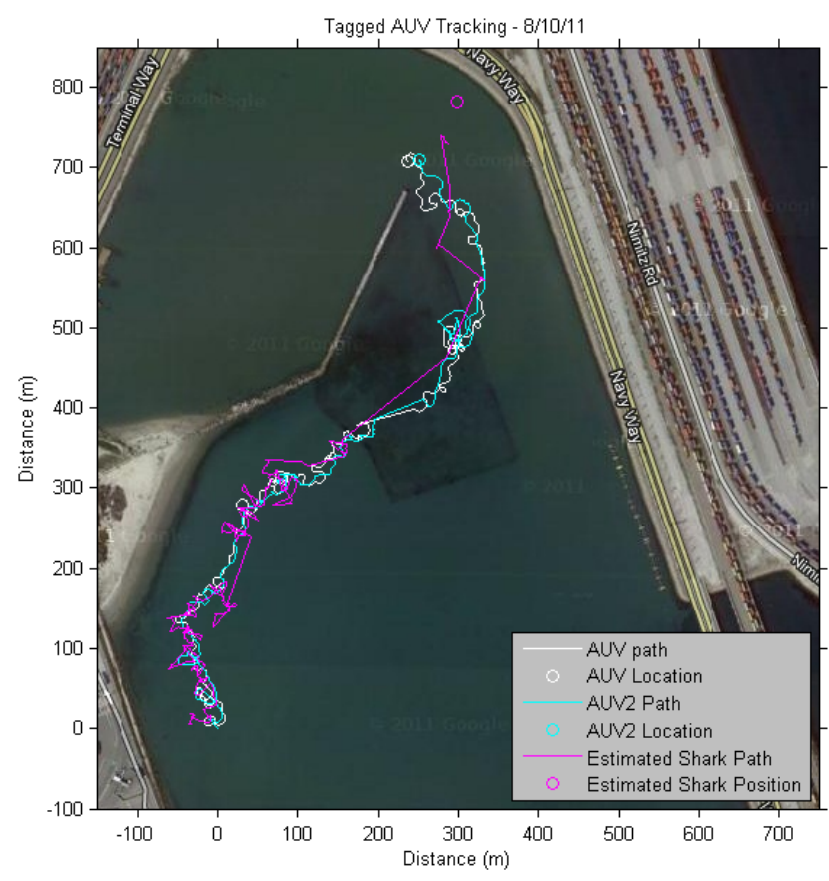

Figure 4.9: Close-up of the Follower AUV, the Secondary AUV, and Estimated Position Trajectories

received a better signal rate. Figure 4.8 presents the cost function and its derivative at each time step. Similar to the stationary tag tracking results, the cost function decreases when the LOTEK signal rate increases and vice versa. Also, the error is defined as the distance between the actual and estimated secondary AUV location:

$$
e_{t}=\sqrt{\left(x_{G P S}-x_{E s t .}\right)^{2}+\left(y_{G P S}-y_{E s t .}\right)^{2}}
$$

\subsubsection{Shark Tracking}

When the results of the stationary tag and AUV tagged tracking were promising, the final phase of the research was planned to be done on a real shark. On August 2011, at SeaPlane Lagoon (located at Long Beach), a 2 meter leopard 
shark was lured and an acoustic transmitter was attached to its fin. Experiments took 3 days. A total of 36 hours of operation were performed by the AUV following the shark. Similar to the other experiments, the results of the experiment are presented in Figures 4.10 and 4.11. In Figure 4.11 the AUV yaw angle and the relative bearing to the tag are plotted in one control sequence. As it is presented, there is a big gap in the relative bearing to the tag plot. This is due to a rapid movement of the shark or a reduction in the LOTEK signal rate which reduces the accuracy of the localizer algorithm. As discussed in the control system chapter, the AUV follows the tag on S-shape paths. The graph of the AUV yaw angle in Figure 4.11 clearly implies this matter. In fact, the AUV yaw angle oscillates around the angle to the shark which provides multiple sensor vantage points. Considering this, there is however overshoot, i.e. greater than 45 degrees difference between the angle to the shark and the yaw angle of the AUV, in the results due to the environmental effects to the AUV maneuverability and constant displacement of the shark. Also, The velocity of the shark is presented in Figure 4.12. There are some jumps in the graph due to a low LOTEK signal rate which reduces the localization accuracy. It is clear that by having more signals localization is performed more accurate. Finally, the average velocity of the shark from the results of this experiment was calculated $1.775 \frac{\mathrm{m}}{\mathrm{s}}$. 


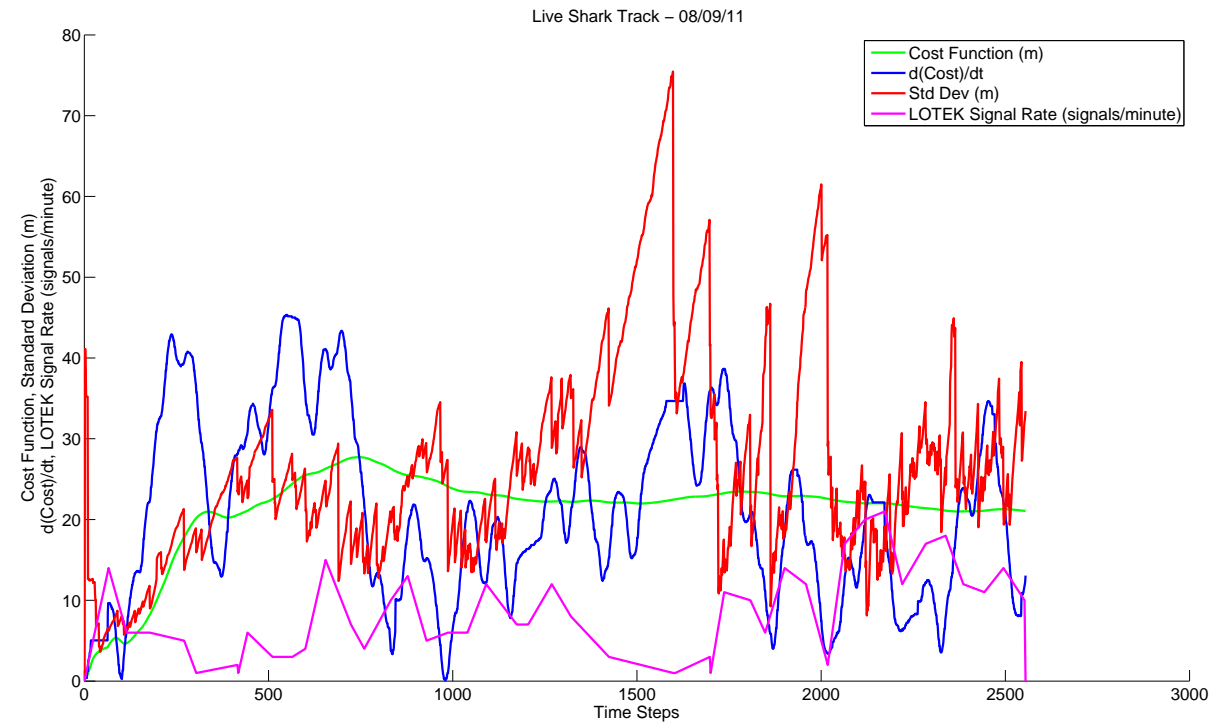

Figure 4.10: Cost Function, Standard Deviation, Cost Function First Derivative, and LOTEK Signal Rate from Tracking a Tagged Shark

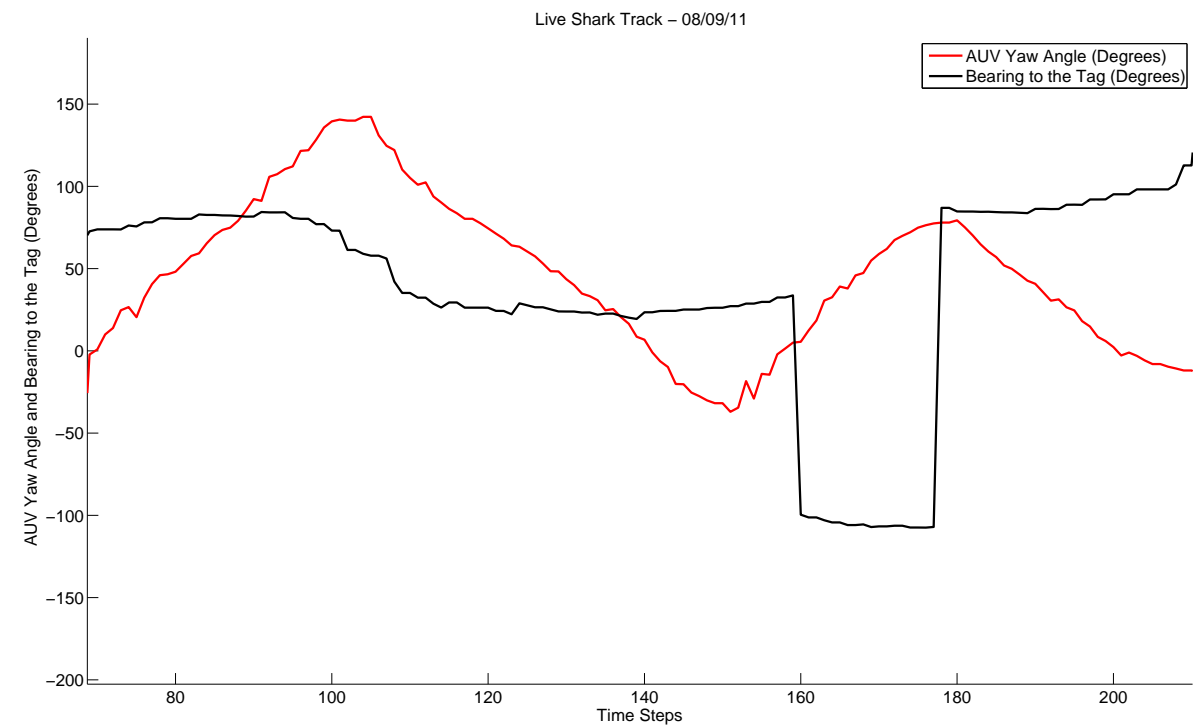

Figure 4.11: AUV Yaw Angle and Bearing to the Tag from Tracking a Tagged Shark in One Control Sequence 


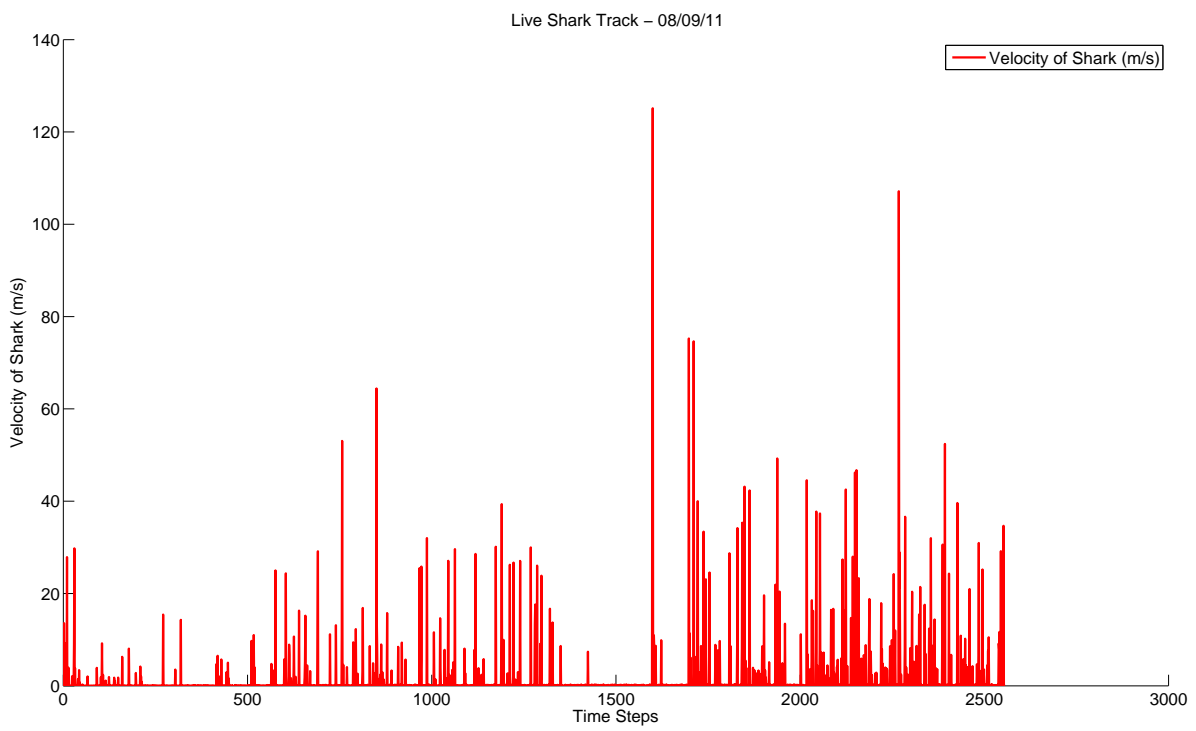

Figure 4.12: Tagged Shark Velocity

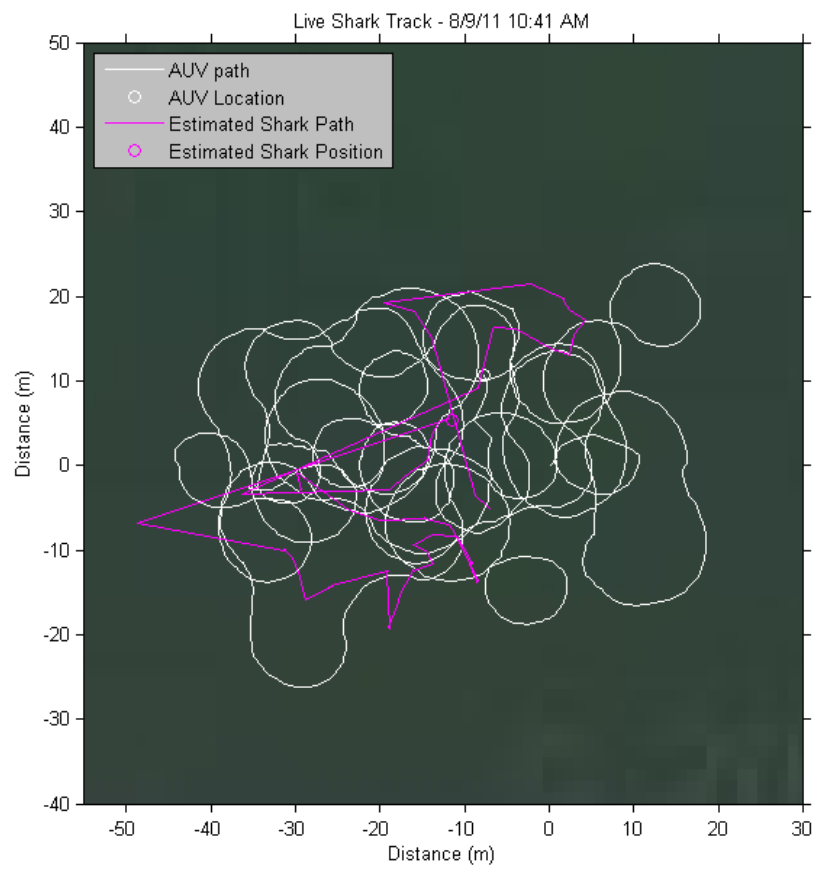

Figure 4.13: Live Shark Path 


\section{Chapter 5}

\section{Conclusion}

In this thesis, an autonomous shark tracking system has been developed. Although the efficiency of the current system is acceptable, there are still improvements to be made. The autonomous tracking system consists of two main parts: 1) A tracking vehicle, and 2) A tracking device. Constraints and criteria for those two parts were investigated and an AUV and an acoustic tracking system were chosen to be used as the tracking vehicle and tracking device. Also, the control system which navigates the AUV to follow a shark was designed. The control system was chosen to be a proportional control system. In parallel, simulations were done to troubleshoot the probable problems during the operations. Based on the simulations, an improved control system was designed which locates the shark faster than the previous one. The improved control system receives the bearing to the shark from the particle filter algorithm. Then it sets the proper fin angles and navigates the AUV to follow the shark. Many experiments including stationary tag tracking, Kayak tracking, and secondary AUV tracking were done to evaluate the accuracy of the system. Finally, in the SeaPlane lagoon the tracking system followed a tagged shark for 1 hour and 41 minutes continuously. 
At this stage, it is required to compare the results from the system with the constraints and criteria which were defined in chapter 2. As discussed before, the maximum sustainable swim speed of Leopard sharks is $0.97 \frac{\mathrm{m}}{\mathrm{s}}$. But the results of the SeaPlane Lagoon experiments, showed the speed value of $1.775 \frac{\mathrm{m}}{\mathrm{s}}$. Although that the calculated value is 1.83 times greater than the maximum sustainable swim speed of Leopard sharks, but still is less than the maximum velocity of the AUV which is $2 \frac{m}{s}$. Therefore the first criteria which required the vehicle have a greater speed than Leopard Sharks average speed is met. For the second criteria, maneuverability of sharks, the result proved that Leopard sharks can change their direction with full speed. There were some situations which the shark had turned 180 degrees with the full speed. On the other hand, mounting frame which was attached to the AUV reduced the angular velocity of the vehicle due to increase of water friction. Without the frame, the AUV can operate with the angular velocity of $0.13 \frac{\mathrm{rad}}{\mathrm{s}}$. With the frame, the angular velocity was reduced to $0.08 \frac{\mathrm{m}}{\mathrm{s}}$. Although the angular velocity was reduced, but from the results it is clearly proven that the maneuverability criteria is met. Finally, response time and detection range of the LOTEK tracking system were recorded as 1 ping per 3 seconds and 150 meters of detection range. 


\section{Chapter 6}

\section{Future Works}

Although operations seemed to be smooth, many limitations exist e.g. inaccuracy of the tracking device, short life of the batteries (which just operate 10 hours continuously), and less AUV maneuverability with the hydrophones attached. For the current tracking device, it is better to have a stronger tag which can propagate stronger signals into the environment. Although the current tracking system can successfully follow a Leopard shark, this might not be true for faster swimming sharks such as great white sharks. Consequently, a larger range would be a big issue for tracking operations. More importantly, this research does not consider obstacles in front of the AUV and assumes that the path has no obstacle. For the next phases of the current research, it is required to satisfy the requirements for tracking other types of fishes. A better tracking device might localize the fish directly, instead of more efforts for localization by the control system, with a wider range of operation. Also, a better thruster would be able to accelerate the AUV with the hydrophones attached more than the current one. Finally, this work promotes the use of collaborative multi-AUV tracking that may improve accuracy and reduce the likelihood of losing the shark. 


\section{Bibliography}

[1] Wikipedia http://en.wikipedia.org/wiki/autonomous-underwater-vehicle.

[2] T. Austin, R. Stokey, C. von Alt, R. Arthur, and R. Goldsborough. 'rats', a relative acoustic tracking system developed for deep ocean navigation. In OCEANS '97. MTS/IEEE Conference Proceedings, volume 1, pages 535 540 vol.1, Oct. 1997.

[3] B. Balasuriya, M. Takai, W. Lam, T. Ura, and Y. Kuroda. Vision based autonomous underwater vehicle navigation: underwater cable tracking. In OCEANS '97. MTS/IEEE Conference Proceedings, volume 2, pages 1418 -1424 vol.2, Oct. 1997.

[4] J. Campana, V.and Knight. Characterization of an dual acoustic receiver system for measuring range and bearing to acoustic tags. Technical report, Cal Poly State University, 2010.

[5] D. Channel. Ocean of fear: Perfect predators. Documentary, 2008.

[6] S. J. Cooke, G. H. Niezgoda, K. C. Hanson, C. D. Suski, F. J. S. Phelan, R. Tinline, and D. P. Philipp. Use of CDMA acoustic telemetry to document 3-d positions of fish: Relevance to the design and monitoring of aquatic protected areas. Marine Technology Society Journal, 39(1):31-41, 2005. 
[7] P. H. Dana. Global positioning system overview. In Department of Geography, The University of Colorado at Boulder, Aug. 2000.

[8] D. Doug, O. Alan, S. Michele, K. Gabriel, and R. Dudley. Acoustic tracking technology and potential applications for salmonid research within the san francisco bay and sacramento-san joaquin delta prepared. In California Urban Water Agencies, Nov. 2003.

[9] Y. Fan and A. Balasuriya. Autonomous target tracking by AUVs using dynamic vision. In Underwater Technology, 2000. UT 00. Proceedings of the 2000 International Symposium on, pages 187 -192, 2000.

[10] Y. Fan and A. Balasuriya. Target tracking by underwater robots. In Systems, Man, and Cybernetics, 2001 IEEE International Conference on, volume 2, pages $696-701$ vol.2, 2001 .

[11] Florida Museum of Natural History: Ichthyology. International shark attack file, Dec. 2010.

[12] C. Forney. State estimation for tracking of tagged sharks with an auv. In Masters Thesis, Department of Computer Science, Califronia Polytechnic State Universtiy, Dec. 2011.

[13] A. Gadre, D. Maczka, D. Spinello, B. McCarter, D. Stilwell, W. Neu, M. Roan, and J. Hennage. Cooperative localization of an acoustic source using towed hydrophone arrays. In Autonomous Underwater Vehicles, 2008. AUV 2008. IEEE/OES, pages 1 -8, Oct. 2008.

[14] C. Georgiades, A. German, A. Hogue, H. Liu, C. Prahacs, A. Ripsman, R. Sim, L.-A. Torres, P. Zhang, M. Buehler, G. Dudek, M. Jenkin, and 
E. Milios. Aqua: an aquatic walking robot. In Intelligent Robots and Systems, 2004. (IROS 2004). Proceedings. 2004 IEEE/RSJ International Conference on, volume 4, pages 3525 - 3531 vol.4, Sept.-2 Oct. 2004.

[15] J. B. Graham, H. Dewar, N. C. Lai, W. R. Lowell, and S. M. Arce. Aspects of shark swimming performance determined using a large water tunnel. Journal of Experimental Biology, 151(1):175-192, 1990.

[16] T. Grey. What Is an Autonomous Underwater Vehicle? http://www.wisegeek.com/what-is-an-autonomous-underwater-vehicle.htm, 2003.

[17] T. Grothues and J. Dobarro. Fish telemetry and positioning from an autonomous underwater vehicle (AUV). In Instrumentation ViewPoint, 2009.

[18] T. Grothues, J. Dobarro, J. Ladd, A. Higgs, G. Niezgoda, and D. Miller. Use of a multi-sensored AUV to telemeter tagged atlantic sturgeon and map their spawning habitat in the Hudson river, USA. In Autonomous Underwater Vehicles, 2008. AUV 2008. IEEE/OES, pages 1 -7, Oct. 2008.

[19] T. M. Grothues. A review of acoustic telemetry technology and a perspective on its diversification relative to coastal tracking arrays. In J. L. Nielsen, N. Fragoso, M. Lutcavage, H. Arrizabalaga, A. Hobday, and J. Sibert, editors, Tagging and Tracking of Marine Animals with Electronic Devices, volume 9 of Reviews: Methods and Technologies in Fish Biology and Fisheries, pages 77-90. Springer Netherlands, 2009. 10.1007/978-1-4020-9640-2-5.

[20] T. M. Grothues, K. W. Able, J. Mcdonnell, and M. M. Sisak. An estuarine observatory for real-time telemetry of migrant macrofauna: Design , performance, and constraints. Observatory, 3:275-289, 2005. 
[21] R. D. Hedger, F. Martin, J. J. Dodson, D. Hatin, F. Caron, and F. G. Whoriskey. The optimized interpolation of fish positions and speeds in an array of fixed acoustic receivers. ICES Journal of Marine Science: Journal du Conseil, 65(7):1248-1259, 2008.

[22] M. R. Heupel, J. M. Semmens, and A. J. Hobday. Automated acoustic tracking of aquatic animals: scales, design and deployment of listening station arrays. 57(1):1-13, Jun. 2006.

[23] M. R. Heupel, C. A. Simpfendorfer, and R. E. Hueter. Estimation of shark home ranges using passive monitoring techniques. Environmental Biology of Fishes, 71(2):135-142, 2004.

[24] J. Irza and M. Desai. Signal processing and data fusion for autonomous undersea vehicles. In Unmanned Untethered Submersible Technology, 1989. Proceedings of the 6th International Symposium on, pages 393 -400, Jun. 1989.

[25] H. Iwakami, T. Ura, K. Asakawa, T. Fujii, Y. Nose, J. Kojima, Y. Shirasaki, T. Asai, S. Uchida, N. Higashi, and T. Fukuchi. Approaching whales by autonomous underwater vehicle. Marine Technology Society Journal, $36(1): 80-85,2002$.

[26] M. Johnson and P. Tyack. A digital acoustic recording tag for measuring the response of wild marine mammals to sound. Oceanic Engineering, IEEE Journal of, 28(1):3 - 12, Jan. 2003.

[27] B. Kalyan, A. Balasuriya, H. Kondo, T. Maki, and T. Ura. Motion estimation and mapping by autonomous underwater vehicles in sea environments. In Oceans 2005 - Europe, volume 1, pages 436 - 441 Vol. 1, Jun. 2005. 
[28] A. Keet. Migration of Sharks. http://www.sharks.org.za/migration-ofsharks.html.

[29] D. W. Kerstetter, J. J. Polovina, and J. E. Graves. Evidence of shark predation and scavenging on fishes equipped with pop-up satellite archival tags. The Free Library, Oct. 2004.

[30] G. L. Lacroix, P. McCurdy, and D. Knox. Migration of atlantic salmon postsmolts in relation to habitat use in a coastal system. Transactions of the American Fisheries Society, 133(6):1455-1471, 2004.

[31] A. Lang. American physical societys division of fluid dynamics (DFD) annual meeting in Long Beach. Technical report, 2010.

[32] J. J. Leonard, A. A. Bennett, C. M. Smith, H. Jacob, and S. Feder. Autonomous underwater vehicle navigation. In MIT Marine Robotics Laboratory Technical Memorandum, 1998.

[33] K.-H. Lin and C.-C. Wang. Stereo-based simultaneous localization, mapping and moving object tracking. In Intelligent Robots and Systems (IROS), 2010 IEEE/RSJ International Conference on, pages 3975 -3980, Oct. 2010.

[34] A. Matos, N. Cruz, A. Martins, and F. Lobo Pereira. Development and implementation of a low-cost lbl navigation system for an auv. In OCEANS '99 MTS/IEEE. Riding the Crest into the 21st Century, volume 2, pages 774 -779 vol.2, 1999.

[35] D. R. Nelson, J. N. McKibben, W. R. Strong, C. G. Lowe, J. A. Sisneros, D. M. Schroeder, and R. J. Lavenberg. An acoustic tracking of a megamouth shark, megachasma pelagios: a crepuscular vertical migrator. Environmental Biology of Fishes, 49:389-399, 1997. 10.1023/A:1007369619576. 
[36] T. F. Norris, M. M. Donald, and J. Barlow. Acoustic detections of singing humpback whales (megaptera novaeangliae) in the eastern north pacific during their northbound migration. The Journal of the Acoustical Society of America, 106(1):506-514, 1999.

[37] OceanServer. Iver2-580-s specifications, Dec. 2010.

[38] D. Odell, K. Hertel, and C. Nielsen. New acoustic systems for AUV tracking, communications, and noise measurement at NSWCCD-ARD, Lake Pend Oreille, Idaho. In OCEANS '02 MTS/IEEE, volume 1, pages 266 - 271 vol.1, Oct. 2002.

[39] R. O'Dor, Y. Andrade, D. Webber, W. Sauer, M. Roberts, M. Smale, and F. Voegeli. Applications and performance of radio-acoustic positioning and telemetry (RAPT) systems. Hydrobiologia, 371-372:1-8, 1998. 10.1023/A:1017006701496.

[40] T. A. C. Ridign, T. E. Dennis, C. L. Stewart, M. M. Walker, and J. C. Montgomery. Tracking fish using 'buoy-based' GPS telemetry. In Marine Ecology Progress Series, volume 377, pages 255-262, Feb. 2009.

[41] J. Rife and S. Rock. Segmentation methods for visual tracking of deep-ocean jellyfish using a conventional camera. Oceanic Engineering, IEEE Journal of, 28(4):595-608, Oct. 2003.

[42] P. Rigby, O. Pizarro, and S. Williams. Towards geo-referenced auv navigation through fusion of usbl and dvl measurements. In OCEANS 2006, pages $1-6$, Sept. 2006.

[43] A. R. Rodgers, R. S. Rempel, and K. F. Abraham. A gps-based telemetry system. Wildlife Society Bulletin, 24:559-566, 1996. 
[44] D. Schulz, W. Burgard, D. Fox, and A. Cremers. Tracking multiple moving objects with a mobile robot. In Computer Vision and Pattern Recognition, 2001. CVPR 2001. Proceedings of the 2001 IEEE Computer Society Conference on, volume 1, pages I-371 - I-377 vol.1, 2001.

[45] D. Schulz, W. Burgard, D. Fox, and A. Cremers. Tracking multiple moving targets with a mobile robot using particle filters and statistical data association. In Robotics and Automation, 2001. Proceedings 2001 ICRA. IEEE International Conference on, volume 2, pages 1665 - 1670 vol.2, 2001.

[46] C. A. Simpfendorfer, M. R. Heupel, and R. E. Hueter. Estimation of shortterm centers of activity from an array of omnidirectional hydrophones and its use in studying animal movements. Canadian Journal of Fisheries and Aquatic Sciences, 59(1):23-32, 2002.

[47] D. W. Sims, E. J. Southall, A. J. Richardson, P. C. Reid, and J. D. Metcalfe. Seasonal movements and behaviour of basking sharks from archival tagging: No evidence of winter hibernation. Marine Ecology Progress Series, 248(Priede 1984):187-196, 2010.

[48] S. Smith and D. Kronen. Experimental results of an inexpensive short baseline acoustic positioning system for auv navigation. In OCEANS '97. MTS/IEEE Conference Proceedings, volume 1, pages 714 -720 vol.1, Oct. 1997.

[49] A. Treptow and A. Zell. Real-time object tracking for soccer-robots without color information. Robotics and Autonomous Systems, 48(1):41 - 48, 2004. European Conference on Mobile Robots (ECMR '03).

[50] T. Ura, R. Bahl, M. Sakata, J. Kojima, T. Fukuchi, J. Ura, K. Mori, 
T. Nakatani, Y. Nose, and H. Sugimatsu. Development of AUV-based system for acoustic tracking of diving sperm whales. In OCEANS '04. MTTS/IEEE TECHNO-OCEAN '04, volume 4, pages 2302 -2307 Vol.4, Nov. 2004.

[51] T. Ura, J. Kojima, T. Nakano, H. Sugimatus, K. Mori, R. Hirotsu, and M. Yanagishawa. Experimental result of AUV-based acoustic tracking system of sperm whales. In OCEANS 2006 - Asia Pacific, pages 1 -5, May 2006.

[52] K. Vickery. Acoustic positioning systems. a practical overview of current systems. In Autonomous Underwater Vehicles, 1998. AUV'98. Proceedings Of The 1998 Workshop on, pages 5-17, Aug. 1998.

[53] K. Vickery. Acoustic positioning systems. new concepts-the future. In $A u$ tonomous Underwater Vehicles, 1998. AUV'98. Proceedings Of The 1998 Workshop on, pages 103 -110, Aug. 1998.

[54] F. A. Voegeli, M. J. Smale, D. M. Webber, Y. Andrade, and R. K. O'Dor. Ultrasonic telemetry, tracking and automated monitoring technology for sharks. Environmental Biology of Fishes, 60:267-282, 2001. 10.1023/A:1007682304720.

[55] C. C. Wang, C. Thorpe, S. Thrun, M. Hebert, and H. Durrant-Whyte. Simultaneous localization, mapping and moving object tracking. The International Journal of Robotics Research, 26(9):889-916, 2007.

[56] D. W. Welch, G. W. Boehlert, and B. R. Ward. POST-the pacific ocean salmon tracking project. Oceanologica Acta, 25(5):243 - 253, 2002.

[57] B. M. Wetherbee, K. N. Holland, C. G. Meyer, and C. G. Lowe. Use of a 
marine reserve in Kaneohe Bay, Hawaii by the giant trevally, caranx ignobilis. Fisheries Research, 67(3):253 - 263, 2004.

[58] M. G. Wing, A. Eklund, and L. D. Kellogg. Consumer-grade global positioning system (gps) accuracy and reliability. Journal of Forestry, 103(4):169$173,2005$.

[59] X. Xu and S. Negahdaripour. Vision-based motion sensing for underwater navigation and mosaicing of ocean floor images. In OCEANS ' 9 '\%. MTS/IEEE Conference Proceedings, volume 2, pages 1412 -1417 vol.2, Oct. 1997.

[60] A. Xydes, M. Moline, C. Lowe, and C. M. Clark. Behavior characterization and particle filter localization of a shovelnose shark. 2009.

[61] J. Zhou and C. Clark. Autonomous fish tracking by ROV using monocular camera. In Computer and Robot Vision, 2006. The 3rd Canadian Conference on, page 68, Jun. 2006. 